\title{
Volterra equations in Banach spaces with completely monotone kernels
}

\author{
Stefano Bonaccorsi and Gertrud Desch
}

\begin{abstract}
We consider a class of infinite delay equations in Banach spaces that models arising in the theory of viscoelasticity, for instance. The equation involves a completely monotone convolution kernel with a singularity at $t=0$ and a sectorial linear spatial operator. Our main goal here is the construction of a semigroup formulation for the integral equation; in the last part of the paper, we illustrate the potentiality of the approach by considering a stochastic perturbation of the problem. Existence and uniqueness of a weak solution is established. The corresponding evolutionary solution process is Markovian, and the tools of linear analytic semigroup theory can be utilized.
\end{abstract}

Mathematics Subject Classification. 60H15, 60H20, 45K05.

Keywords. Abstract integrodifferential equation, Random forcing term, Singular kernel, Stationary state, Analytic semigroup.

\section{Introduction}

In this paper we consider a Volterra integrodifferential equation of the form:

$$
\begin{aligned}
\frac{d}{d t} \int_{-\infty}^{t} a(t-s) u(s) \mathrm{d} s & =A u(t)+f(t) \quad \text { for } t \in[0, \infty) \\
u(t) & =u_{0}(t) \quad \text { for } t \leq 0
\end{aligned}
$$

in a Banach space $(E,|\cdot|)$. Here $-A$ is a linear sectorial operator on $E$ and $a$ is a scalar kernel, completely monotonic and locally integrable with $a(0+)=\infty$. The task of the paper is to develop a semigroup approach to (1.1). In the second part of the paper this approach will be applied to treat a Volterra integrodifferential equation with stochastic forcing 


$$
\begin{aligned}
\frac{d}{d t} \int_{-\infty}^{t} a(t-s) u(s) \mathrm{d} s & =A u(t)+\Phi(t) \frac{d}{d t} W(t) \quad \text { for } t \in[0, \infty) \\
u(t) & =u_{0}(t) \quad \text { for } t \leq 0
\end{aligned}
$$

Here, $W(t)$ is a cylindrical Wiener process with values in a Hilbert space $U$, and $\Phi$ takes values in $\gamma(U ; E)$, the space of $\gamma$-radonifying operators from $U$ to $E$.

Existence, uniqueness, and regularity of solutions of (1.1) and (1.2) have been handled successfully by the highly developed theory of resolvent operators (see, e.g., [22], also [11,29] for more recent developments on regularity). Volterra equations with stochastic forcing have been investigated for a decade, just to mention a few, $[3,4,6-9,13,14]$. These papers generally combine resolvent operators with the machinery of stochastic convolutions (see e.g. [12]). Thus, from the viewpoint of existence, uniqueness and regularity questions, deterministic and stochastic Volterra integrodifferential equations can be handled very efficiently by more convenient methods than the somewhat bulky semigroup approach.

However, there are questions which do require some kind of state space approach. To explain the concept of a state space notice that (1.1) and (1.2) are equations with memory. The future of the system does not exclusively depend on the present value of the solution $u$, but information from the history of $u$ is passed on to the future by the integral term. Therefore, any questions concerning equilibria, controllability, optimal feedback control, etc., cannot be answered without regard to the past. In the stochastic case this implies that $u$ is not a Markov process. Again, considerations about stationary distributions or transition probabilities make only sense if the past is included. To consider the history in an appropriate way, one may construct a state space. Roughly speaking, the state of a system contains all information which is relevant for the further evolution. Thus, by construction, further development of the system depends only on the present value of the state.

In our setting, the integral equations (1.1), (1.2) are rewritten to differential equations for the state $v(t)$ in some large space $X$ :

$$
\begin{aligned}
\frac{d}{d t} v(t) & =B v(t)+(1-B) P f(t), \\
\mathrm{d} v(t) & =B v(t) \mathrm{d} t+(1-B) P \Phi(t) \mathrm{d} W(t),
\end{aligned}
$$

respectively, with suitable linear operators $B$ and $P$. The solution $u$ is recovered from the state by an operator $u(t)=J v(t)$, and the initial state $v(0)$ is constructed from the initial function: $v(0)=Q u_{0}$. In our setting, $B$ generates an analytic semigroup in the state space. Now we can utilize the impressive toolbox of analytic semigroups, in particular the regularity theory using interpolation spaces [21]. Though $B$ and $J$ are not bounded operators, these tools allow to make sense of (1.3). In the stochastic case, we can resort to the concept of stochastic convolution, developed for Hilbert spaces in [12]. To go beyond Hilbert spaces and develop an $\mathbf{L}^{p}$-theory, we take advantage of recent developments on stochastic integration in UMD-spaces and their applications to stochastic differential equations (see $[25,26]$ and references therein). 
Our state space goes back to [20], motivated by Ph. Clément, who proposed to adapt a semigroup approach for linear viscoelasticity [15] to the parabolic problem (1.1) and its stochastic counterpart. The results from [20] include a state space theory for scalar valued stochastic Volterra equations, and for Hilbert space valued equations with self-adjoint operator $A$, where spectral resolution can be applied. These results were extended by [10] (deterministic) and [2] (stochastic) to more general operators in Hilbert spaces. The state space approach has already proved to be useful for treating control problems $[1,5]$. In the present paper we will provide simplified proofs and generalize the theory to reflexive Banach spaces. For stochastic problems we require UMD-spaces of type 2 .

As an application of the semigroup setting proposed here, we provide a result of existence and uniqueness for the solution of the deterministic equation (1.1), in Theorem 2.7, and for the stochastic equation (1.2), in Theorem 2.14. Moreover, Sect. 6 is devoted to discuss the transition semigroup for the solution of the state space stochastic evolution equation associated to (1.2). We give sufficient conditions for the solution process to admit an invariant measure, in Theorem 6.2, and we discuss how this property can be translated in terms of the original Volterra equation.

\section{Statement of the main results}

Since the construction of the state space is quite technical and lengthy, we will first summarize the results of this paper and defer the proofs to the subsequent sections.

\subsection{The deterministic equation}

Throughout this paper let $(E,|\cdot|)$ be a reflexive Banach space. $p$ and $q$ are conjugate exponents, i.e., real positive constants such that $\frac{1}{p}+\frac{1}{q}=1$. By $\Sigma(\omega)$ we denote the sector

$$
\Sigma(\omega)=\{z \in \mathbb{C} \backslash\{0\}|| \arg (z) \mid<\omega\} .
$$

Hypothesis 2.1. We let $A: D(A) \subset E \rightarrow E$ be a linear operator such that $-A$ is sectorial with angle $\omega_{A}$.

As explained in $\left[19\right.$, p. 19], Hypothesis 2.1 says that for $\omega_{A} \in(0, \pi)$,

(1) The cone $\Sigma\left(\pi-\omega_{A}\right)$ is contained in the resolvent set of $A$;

(2) For all $\varepsilon>0$ there exists $M_{\varepsilon}>0$ such that

$$
\forall \lambda \in \Sigma\left(\pi-\omega_{A}-\varepsilon\right): \quad\left\|\lambda(A-\lambda)^{-1}\right\| \leq M_{\varepsilon} .
$$

Notice also that such $A$ is densely defined by [19, Proposition 2.1.1.(h)]

Notation 2.2. As usual, we denote the resolvent $R(s, A):=(s-A)^{-1}$.

For $\theta \in(0,1)$ we consider interpolation and extrapolation with respect to $A$ : 
(a) $E_{1}=D(A)$ equipped with the graph norm of $A$.

(b) $E_{\theta, p}=\left(E, E_{1}\right)_{(\theta, p)}$ which means the real interpolation space between $E$ and $E_{1}$.

(c) $E_{-1}$ is the completion of $E$ with respect to the norm $|x|_{-1}=\mid(A-$ $\omega)\left.(x)\right|_{E}$. (Here all $\omega>0$ yield the same space, up to equivalence of norms.)

(d) $E_{\theta-1, p}=\left(E_{-1}, E\right)_{(\theta, p)}$ in the sense of real interpolation.

The norm in the interpolation space $E_{\mu, p}$ will be denoted by $|\cdot|_{\mu, p}$.

The key point of our construction is the kernel $a(t)$. Properties of completely monotone kernels can be found in many classical titles as, e.g., the monograph [18]. Remember that a completely monotone function $a:(0, \infty) \rightarrow$ $(0, \infty)$ is a function such that for all $k \in \mathbb{N}$ and all $t>0$

$$
(-1)^{k} \frac{d^{k}}{d t^{k}} a(t)>0 .
$$

By Bernstein's Theorem, $a$ is completely monotone if and only if there exists a (nonnegative) measure $\nu$ on $[0, \infty)$ such that for all $t>0$

$$
a(t)=\int_{[0, \infty)} e^{-\kappa t} \nu(\mathrm{d} \kappa) .
$$

As usual we denote the Laplace transform of $a$ by $\hat{a}$, and we have

$$
\hat{a}(s)=\int_{[0, \infty)} \frac{1}{s+\kappa} \nu(\mathrm{d} \kappa) .
$$

Definition 2.3. To any completely monotone kernel $a(t)$ we associate a constant, denoted $\alpha(a)$, defined as the supremum of all $\alpha \in(0,1)$ such that $\liminf _{t \rightarrow 0+} t^{\alpha} a(t)>0$.

Hypothesis 2.4. We assume that $a:(0, \infty) \rightarrow(0, \infty)$ is a completely monotone kernel with associated Bernstein measure $\nu$, such that

(1) $\int_{0}^{1} a(t) \mathrm{d} t<+\infty$;

(2) $a(0+)=+\infty$;

(3) there exists some $\omega_{a} \in\left(0, \pi-\omega_{A}\right)$, such that for all $s \in \mathbb{C}^{+}$we have $s \hat{a}(s) \in \Sigma_{\omega_{a}}$;

(4) the constant $\alpha(a)$ introduced in Definition 2.3 satisfies $\alpha(a) \in(0,1)$.

Some consequences of the above assumptions, that will be often used in the sequel, are collected in the following remark.

Remark 2.5. The following assertions follow immediately from Hypothesis 2.4:

(1) $\int_{[0, \infty)}(\kappa+1)^{-1} \nu(\mathrm{d} \kappa)<+\infty$ (equivalent to Hypothesis 2.4(1)),

(2) $\nu([0, \infty))=+\infty$ (equivalent to Hypothesis 2.4(2)),

(3) $\liminf _{s \rightarrow \infty} s^{1-\alpha(a)} \hat{a}(s)>0$ (from Hypothesis 2.4(4)). 
Under these hypotheses we investigate existence, uniqueness and regularity results for the solution to the deterministic integral equation (1.1), i.e.,

$$
\begin{gathered}
\frac{d}{d t} \int_{-\infty}^{t} a(t-s) u(s) \mathrm{d} s=A u(t)+(t), \\
\quad(\forall s<0 \text { a.e. }) \quad u(s)=u_{0}(s) .
\end{gathered}
$$

We will resort to the concept of integrated solutions.

Definition 2.6. We assume that Hypotheses 2.1 and 2.4 hold. Let $f \in$ $\mathbf{L}_{l o c}^{1}([0, \infty) ; E)$ and $u_{0} \in \mathbf{L}^{1}((-\infty, 0] ; E)$ with $\int_{0}^{\infty} a(\tau)\left|u_{0}(-\tau)\right| \mathrm{d} s<+\infty$.

We say that $u \in \mathbf{L}_{l o c}^{1}((-\infty, \infty) ; E)$ is an integrated solution of (1.1) if and only if the following properties hold:

$$
\begin{aligned}
& (\forall t \leq 0 \text { a.e. }) \quad u(t)=u_{0}(t), \\
& (\forall t \geq 0) \quad \int_{0}^{t} u(\tau) \mathrm{d} \tau \in D(A), \quad \text { and } \\
& \int_{-\infty}^{t} a(t-\tau) u(\tau) \mathrm{d} \tau-\int_{-\infty}^{0} a(-\tau) u_{0}(\tau) \mathrm{d} \tau=A \int_{0}^{t} u(\tau) \mathrm{d} \tau+\int_{0}^{t} f(\tau) \mathrm{d} \tau .
\end{aligned}
$$

To motivate our choice of the state space, we propose the following computation:

$$
\begin{aligned}
\int_{-\infty}^{t} a(t-s) u(s) \mathrm{d} s & =\int_{[0, \infty)} \int_{-\infty}^{t} e^{-\kappa(t-s)} u(s) \mathrm{d} s \nu(\mathrm{d} \kappa)=\int_{[0, \infty)} v(t, \kappa) \nu(\mathrm{d} \kappa) \\
\text { with } \quad v(t, \kappa) & =\int_{-\infty}^{t} e^{-\kappa(t-s)} u(s) \mathrm{d} s
\end{aligned}
$$

Thus, (1.1) translates into

$$
\frac{\partial}{\partial t} \int_{[0, \infty)} v(t, \kappa) \nu(\mathrm{d} \kappa)=A u(t)+f(t),
$$

while $v(t, \kappa)$ satisfies, at least formally, the differential equation

$$
\frac{\partial}{\partial t} v(t, \kappa)=-\kappa v(t, \kappa)+u(t)
$$

and we can impose the initial condition

$$
v_{0}(\kappa):=v(0, \kappa)=\int_{-\infty}^{0} e^{\kappa s} u_{0}(s) \mathrm{d} s .
$$

Our idea is, consequently, to construct a suitable space $X$ of functions $v:[0, \infty) \rightarrow E$ and to consider $v(t)=v(t, \cdot)$ as the state of the system. Then (2.1) und (2.2) can be rewritten as an abstract differential equation in $X$ 


$$
\frac{d}{d t} v(t)=B v(t)+(1-B) P f(t), \quad v(0)=v_{0}
$$

with suitable linear operators $B: D(B) \subset X \rightarrow X$ and $P: E \rightarrow X$. The initial state is given by $v_{0}=Q u_{0}$, where the operator $Q: \mathbf{L}^{1}((-\infty, 0] ; E) \rightarrow$ $\mathbf{L}^{\infty}([0, \infty) ; E ; \mathrm{d} \nu)$ is constructed by (2.3). Finally, the function $u$ can be recovered by $u(t)=J v(t)$, with a linear operator $J: D(J) \subset X \rightarrow E$, essentially by means of equation (2.2) and the constraint (2.1).

We will show that $B$ is the generator of an analytic semigroup in $X$, so that we can define the semigroup solution to (2.4) by

$$
v(t)=e^{t B} v_{0}+\int_{0}^{t}(1-B) e^{(t-\tau) B} \operatorname{Pf}(\tau) \mathrm{d} \tau .
$$

Similarly as in Notation 2.2 we let $X_{\theta, p}$ denote the real interpolation and extrapolation spaces of $X$ with respect to $B$ and $\|\cdot\|_{\theta, p}$ their norms.

The following theorem gives the relation between the abstract state space and Problem (1.1).

Theorem 2.7. We assume that Hypotheses 2.1 and 2.4 hold. Let $X, B, P, J, Q$ be defined by Definitions 3.1, 3.4, 3.6, 3.7, 3.10. Let $\eta$ and $\theta$ be such that

$$
\frac{1}{p}-\frac{\alpha(a)}{p}<\eta<\frac{1}{p}, \quad \eta<\theta<\frac{1}{p}+\frac{\alpha(a)}{q} .
$$

Let $u_{0} \in \mathbf{L}^{1}((-\infty, 0] ; E)$ with $\int_{0}^{\infty} a(\tau)\left|u_{0}(-\tau)\right| \mathrm{d} \tau<+\infty$ and let $f \in$ $\mathbf{L}_{l o c}^{1}([0, \infty) ; E)$.

Set $v_{0}=Q u_{0}$. If $v$ given by (2.5) is the solution in semigroup sense of the abstract Cauchy problem (2.4), then the following assertions hold:

(1) $v_{0} \in X_{-\frac{1}{q}, p}$, the operator $P$ maps $E$ continuously into $X_{\theta, p}$ and $J$ maps $X_{\eta, p}$ continuously into $E$.

(2) $v(t) \in \mathbf{L}_{l o c}^{1}\left([0, \infty) ; X_{\eta, p}\right)$, thus $J v(t)$ is well defined almost everywhere, and $J v \in \mathbf{L}_{l o c}^{1}([0, \infty) ; E)$.

(3) The function $u:(-\infty, T] \rightarrow E$, defined by

$$
u(t)= \begin{cases}u_{0}(t) & \text { if } t \leq 0 \\ J v(t) & \text { if } t>0\end{cases}
$$

is the unique integrated solution of (1.1).

The construction of the state space and the operators $B, J, P, Q$ will be carried out in Sect. 3. The connection of the state space setting to (1.1) and the proof of Theorem 2.7 will then follow in Sect. 4. In Sect. 4 we will also give some further regularity results (Corollaries 4.2 and 4.3).

\subsection{The stochastic equation}

To treat the stochastic problem (1.2), we need some additional assumptions:

Again we have a Banach space $(E,|\cdot|)$ which is now required to be a UMD-space of type 2.

$p$ and $q$ are conjugate exponents with

$$
1<q \leq 2 \leq p<\infty .
$$


The operator $A$ satisfies Hypothesis 2.1, and the convolution kernel $a$ satisfies Hypothesis 2.4 with the additional requirement that

$$
\alpha(a)>\frac{1}{2}
$$

Remark 2.8. We give a short reason for these requirements:

For stochastic integration in the Banach space $E$, we require that $E$ is UMD and has type 2 , and that $p \geq 2$, so that $L^{p}(M ; E)$ inherits these properties from $E$ for any measure space $M$.

Sufficient singularity of the kernel $a$ is required to ensure that the stochastic forcing is sufficiently regularized by the analytic semigroup $e^{t B}$. Choose $\eta, \theta$ according to Theorem 2.7, so that $J: X_{\eta, p} \rightarrow E$ and $P: E \rightarrow X_{\theta, p}$ are continuous. This requires

$$
\frac{1}{p}-\frac{\alpha(a)}{p}<\eta<\theta<\frac{1}{p}+\frac{\alpha(a)}{q},
$$

so $\alpha(a)>\theta-\eta$. We will need that the stochastic convolution

$$
\int_{0}^{t}(1-B) e^{(t-s) B} P \Phi(s) \mathrm{d} W(s)
$$

takes values in $X_{\eta, p}$. This requires that $\theta-\eta>\frac{1}{2}$ which can hold only if (2.8) is satisfied.

We impose the following assumptions on the stochastic processes:

Hypothesis 2.9. (Probability space and Wiener process) $(\Omega, \mathcal{F}, \mathbb{P})$ is a probability space with a filtration $\left\{\mathcal{F}_{t}\right\}$. Let $U$ be a real separable Hilbert space. Let $W$ be an isonormal Gaussian process (a white noise) on $L^{2}\left(\mathbb{R}_{+} ; U\right)$, i.e.,

(1) the random variables $W\left(h_{1}\right), \ldots W\left(h_{n}\right)$ are jointly mean zero Gaussian for all $h_{1}, \ldots, h_{n} \in L^{2}\left(\mathbb{R}_{+} ; U\right)$, and

(2) for $h, g \in L^{2}\left(\mathbb{R}_{+} ; U\right)$ the covariance is given by $\mathbb{E}[W(g) \cdot W(h)]=$ $\langle g, h\rangle_{L^{2}\left(\mathbb{R}_{+} ; U\right)}$.

In the following hypothesis, as usual, we denote by $\gamma(U ; Y)$ the space of $\gamma$-radonifying operators from $U$ into a Banach space $Y$. The concept of $\gamma$-radonifying operators and stochastic integration in Banach spaces will be shortly exposed in Sect. 5.1.

Hypothesis 2.10. (The covariance operator) We assume that either one of the following conditions holds (depending on whether $\Phi$ depends on $t$ or not):

(1) let $p>\frac{2}{2(\theta-\eta)-1}$ and suppose that $\Phi(t)$ is a stochastic process with values in $L_{\mathcal{F}}^{p}\left(\Omega ; L^{2}\left(\mathbb{R}_{+} ; \gamma(U, E)\right)\right.$ and for some $\delta$ with $\frac{1}{p}+1-(\theta-\eta)<\delta<\frac{1}{2}$ it holds: 
$\forall T \quad \exists C=C_{T} \quad$ such that $\sup _{t \in[0, T]} \mathbb{E}\left|\int_{0}^{t}\left\|(t-r)^{-\delta} \Phi(r)\right\|_{\gamma(U, E)}^{2} \mathrm{~d} r\right|^{p / 2} \leq C_{T}$.

(2) $\Phi(t):=\Phi$ is constant with a $\gamma$-Radonifying operator $\Phi \in \gamma(U, E)$.

Remark 2.11. In case Hypothesis 2.10(2) holds, condition (2.9) is obviously satisfied for any $p$.

With these assumptions we consider a class of stochastic Volterra equation (1.2), that arises from problem (1.1) when we introduce a random forcing term:

$$
\begin{aligned}
\frac{d}{d t} \int_{-\infty}^{t} a(t-s) u(s) \mathrm{d} s & =A u(t)+\Phi(t) \frac{d}{d t} W(t) \quad \text { for } t \in[0, \infty) \\
u(t) & =u_{0}(t) \quad \text { for } t \leq 0 .
\end{aligned}
$$

Definition 2.12. We assume that Hypothesis 2.1, 2.4, 2.9 and 2.10 hold. Let $\{u(t)\}_{t \geq 0}$ be an adapted stochastic process with values in $E$, such that the following integrability condition holds:

$$
\exists \epsilon>0 \quad \text { such that } \mathbb{E} \int_{0}^{\infty}\|u(t)\|_{E}^{2} e^{-\epsilon t} \mathrm{~d} t<+\infty .
$$

Then $u$ is a weak solution of problem (1.2) if the following equality holds for any $\zeta \in D\left(A^{*}\right)$, almost surely:

$$
\int_{-\infty}^{t}\langle a(t-s) u(s), \zeta\rangle \mathrm{d} s=\langle\bar{u}, \zeta\rangle+\int_{0}^{t}\left\langle A^{*} \zeta, u(s)\right\rangle \mathrm{ds}+\int_{0}^{\mathrm{t}}\langle\zeta, \Phi(\mathrm{s}) \mathrm{dW}(\mathrm{s})\rangle,
$$

where

$$
\bar{u}=\int_{-\infty}^{0} a(-s) u_{0}(s) \mathrm{d} s
$$

(Here, $\langle x, \zeta\rangle$ denotes the action of $\zeta \in E^{*}$ on $x \in E$.)

We choose a comparatively small class of initial processes. Extension to larger classes are possible if one allows for solution process not depending continously on time at $t=0$.

Definition 2.13. For every $\mu \in(0,1)$ and $\delta \in(1,2)$ we let $\tilde{X}_{\mu, \delta}$ be the space of functions $u_{0}:(-\infty, 0] \rightarrow E$ such that $u_{0}(0) \in E_{\mu, p}$ and

$$
\begin{gathered}
\int_{0}^{\infty}\left|u_{0}(-\tau)\right| \mathrm{d} \tau<\infty, \quad \int_{0}^{\infty} a(\tau)\left|u_{0}(-\tau)\right| \mathrm{d} \tau<\infty, \\
\int_{0}^{\infty} \tau^{-\delta}\left|u_{0}(-\tau)-u_{0}(0)\right| \mathrm{d} \tau<\infty
\end{gathered}
$$

Then, we are ready to state the main result of this section, namely the existence (and uniqueness) of a solution to the stochastic integral equation (1.2). 
Theorem 2.14. Let $E$ be a type 2 UMD-space, $p \in[2, \infty)$. We assume that Hypotheses 2.1, 2.4 (with $\alpha(a)>\frac{1}{2}$ ), 2.9 and 2.10 hold. Let $u_{0} \in$ $L^{2}\left(\Omega, \mathcal{F}_{0} ; \tilde{X}_{\mu, \delta}\right)$ with $\mu>\frac{1}{p}$ and $\delta>2-\alpha(a)$.

(1) There exist $0<\eta<\theta<1$ such that $P: E \rightarrow X_{\theta, p}, J: X_{\eta, p} \rightarrow E$, and $Q: \tilde{X}_{\mu, \delta} \rightarrow X_{\eta, p}$ are continuous, and $\theta-\eta>\frac{1}{2}$.

(2) Define $v_{0}=Q u_{0}$. Given the process

$$
v(t)=e^{t B} v_{0}+\int_{0}^{t} e^{(t-s) B}(1-B) P \Phi(s) \mathrm{d} W(s),
$$

we define the process

$$
u(t)= \begin{cases}J v(t), & t>0 \\ u_{0}(t), & t \leq 0\end{cases}
$$

Then $u(t)$ is the unique weak solution to problem (1.2).

The proof of this theorem will be given in Sect. 5 .

\section{The state space}

\subsection{State space and semigroup}

In this subsection we define the state space and the pertinent operators. We arrive at a construction of a linear semigroup.

Definition 3.1. Let $X$ be the Banach space of measurable functions $w$ : $[0, \infty) \rightarrow E$ such that

$$
\|w\|^{p}:=\int_{[0, \infty)}(1+\kappa)^{p-1}|w(\kappa)|^{p} \nu(\mathrm{d} \kappa)<+\infty .
$$

Lemma 3.2. Assume Hypothesis 2.4. Then the space $X$ defined above is continuously embedded in $\mathbf{L}^{1}([0, \infty) ; E ; \mathrm{d} \nu)$.

Proof. We estimate

$$
\begin{aligned}
\int_{[0, \infty)}|w(\kappa)| \nu(d \kappa) & =\int_{[0, \infty)}(1+\kappa)^{-1 / q}(\kappa+1)^{1 / q}|w(\kappa)| \nu(d \kappa) \\
\leq & {\left[\int_{[0, \infty)}(1+\kappa)^{-1} \nu(d \kappa)\right]^{1 / q} } \\
& \times\left[\int_{[0, \infty)}(1+\kappa)^{p-1}|w(\kappa)|^{p} \nu(d \kappa)\right]^{1 / p}
\end{aligned}
$$

The first integral is bounded by Hypothesis 2.4(1), the second integral is $\|w\|$.

Lemma 3.3. For $w \in X$ there exists at most one $u \in E$ such that

$$
\int_{[0, \infty)}(\kappa+1)^{p-1}|\kappa w(\kappa)-u|^{p} \nu(\mathrm{d} \kappa)<+\infty .
$$


Proof. Suppose that $u_{1}, u_{2}$ satisfy $\left\|\kappa w-u_{i}\right\|^{p}<+\infty$. Then by Minkowski's inequality

$$
\int_{[0, \infty)}(\kappa+1)^{p-1}\left|u_{1}-u_{2}\right|^{p} \nu(d \kappa)<+\infty .
$$

Now, Hypothesis 2.4(2) implies that $\left|u_{1}-u_{2}\right|=0$.

The above lemmas imply that the following definition makes sense.

Definition 3.4. We define operators $\tilde{B}: D(\tilde{B}) \subset X \rightarrow X$ and $\tilde{J}: D(\tilde{B}) \rightarrow E$ by $D(\tilde{B})=\left\{w \in X: \exists u \in E\right.$ such that $\left.\int_{[0, \infty)}(\kappa+1)^{p-1}|\kappa w(\kappa)-u|^{p} \nu(d \kappa)<+\infty\right\}$ and, respectively,

$$
\begin{aligned}
{[\tilde{B}(w)](\kappa) } & :=-\kappa w(\kappa)+u, \\
\tilde{J}(w) & :=u .
\end{aligned}
$$

Lemma 3.5. Let Hypotheses 2.1 and 2.4 hold. Let $v \in X, h \in E$ and $s \in \mathbb{C}^{+}$. Then there exists a unique $w \in D(\tilde{B})$ such that

$$
\left\{\begin{array}{l}
(s-\tilde{B}) w=v, \\
u:=\tilde{J} w \in D(A), \\
\int_{[0, \infty)}[\tilde{B} w](\kappa) \nu(\mathrm{d} \kappa)=A u+h .
\end{array}\right.
$$

The solution to (3.1) can be given explicitely by

$$
\begin{aligned}
& u=R(s \hat{a}(s), A)\left[\int_{[0, \infty)} \frac{\kappa}{s+\kappa} v(\kappa) \nu(\mathrm{d} \kappa)+h\right], \\
& w(\kappa)=\frac{1}{s+\kappa}(v(\kappa)+u) .
\end{aligned}
$$

For all $\epsilon>0$ there exists a constant $c=c(\epsilon)$, dependent only on $A, a, p$ and $\epsilon$ such that for all $s \in \mathbb{C}^{+}$with $|s| \geq \epsilon$ we have the estimates

$$
\begin{aligned}
|u| & \leq c\left(|s|^{-1}|\hat{a}(s)|^{-1 / p}\|v\|+|s|^{-1}|\hat{a}(s)|^{-1}|h|\right) \\
& \leq c\left(|s|^{-1 / q-\alpha(a) / p}\|v\|+|s|^{-\alpha(a)}|h|\right), \\
\|w\| & \leq c\left(|s|^{-1}\|v\|+|s|^{-1}|\hat{a}(s)|^{-1 / q}|h|\right) \\
& \leq c\left(|s|^{-1}\|v\|+|s|^{-1 / p-\alpha(a) / q}|h|\right) .
\end{aligned}
$$

Proof. We notice first that by Hypothesis 2.4(3) for $s \in \mathbb{C}^{+}$we have that $R(s \hat{a}(s), A)$ exists and $\|s \hat{a}(s) R(s \hat{a}(s), A)\| \leq c$.

Given $v \in X, h \in E$, we solve (3.1). This says that we have to find $w \in X$ and $u \in D(A)$ such that 
Vol. 20 (2013)

$$
\begin{aligned}
& (s+\kappa) w(\kappa)-u=v(\kappa) \\
& \int_{[0, \infty)}(u-\kappa w(\kappa)) \nu(d \kappa)=A u+h .
\end{aligned}
$$

The first equation yields

$$
w(\kappa)=\frac{1}{s+\kappa}(u+v(\kappa)) .
$$

Using this in the second equation, we obtain

$$
\begin{aligned}
A u+h= & \int_{[0, \infty)}\left[u-\frac{\kappa}{s+\kappa}(u+v(\kappa))\right] \nu(\mathrm{d} \kappa) \\
= & \int_{[0, \infty)} \frac{s}{s+\kappa} \nu(\mathrm{d} \kappa) u-\int_{[0, \infty)} \frac{\kappa}{s+\kappa} v(\kappa) \nu(\mathrm{d} \kappa)=s \hat{a}(s) u \\
& -\int_{[0, \infty)} \frac{\kappa}{s+\kappa} v(\kappa) \nu(\mathrm{d} \kappa) .
\end{aligned}
$$

Consequently

$$
(s \hat{a}(s)-A) u=h+\int_{[0, \infty)} \frac{\kappa}{s+\kappa} v(\kappa) \nu(\mathrm{d} \kappa) .
$$

We have thus proved that (3.1) has at most one solution which is given by (3.2).

Now we have to show that (3.2) does yield a solution. First notice that the integral in (3.2) exists:

$$
\begin{aligned}
\int_{[0, \infty)} \frac{\kappa}{|\kappa+s|}|v(\kappa)| \nu(\mathrm{d} \kappa) \leq & \int_{[0, \infty)}\left|\frac{\kappa}{(\kappa+1)^{1 / q}(\kappa+s)}\right|\left|(\kappa+1)^{1 / q} v(\kappa)\right| \nu(\mathrm{d} \kappa) \\
\leq & \int_{[0, \infty)}\left|\frac{\kappa}{(\kappa+1)^{1 / q}(\kappa+s)^{1 / p}} \frac{1}{(\kappa+s)^{1 / q}}\right| \\
& \times\left|(\kappa+1)^{1 / q} v(\kappa)\right| \nu(\mathrm{d} \kappa) \\
\leq & \int_{[0, \infty)}|\kappa+s|^{-1 / q}\left|(\kappa+1)^{1 / q} v(\kappa)\right| \nu(\mathrm{d} \kappa) \\
\leq & {\left[\int_{[0, \infty)}|\kappa+s|^{-1} \nu(\mathrm{d} \kappa)\right]^{1 / q} } \\
& \times\left[\int_{[0, \infty)}(\kappa+1)^{p-1}|v(\kappa)|^{p} \nu(\mathrm{d} \kappa)\right]^{1 / p} \\
\leq & {\left[\int_{[0, \infty)} 2(\kappa+|s|)^{-1} \nu(\mathrm{d} \kappa)\right]^{1 / q} } \\
& \times\left[\int_{[0, \infty)}(\kappa+1)^{p-1}|v(\kappa)|^{p} \nu(\mathrm{d} \kappa)\right]^{1 / p} \\
\leq & {[2 \hat{a}(|s|)]^{1 / q}\|v\| . }
\end{aligned}
$$


In the last step we have used that $|\kappa+s| \geq \frac{1}{2}(\kappa+|s|)$ for $\kappa>0$ and $s \in \mathbb{C}^{+}$. We have therefore that

$|u|=\left|R(s \hat{a}(s), A)\left(\int_{[0, \infty)} \frac{\kappa}{\kappa+s} v(\kappa) \nu(\mathrm{d} \kappa)+h\right)\right| \leq \frac{c}{|s \hat{a}(s)|}\left[2 \hat{a}(|s|)^{1 / q}\|v\|+|h|\right]$.

Since $|\kappa+s| \geq|s|$ for $\kappa>0, s \in \mathbb{C}^{+}$, it is evident that $\frac{1}{\kappa+s} v \in X$ with $\left\|\frac{1}{\kappa+s} v\right\| \leq \frac{1}{|s|}\|v\|$.

If $|s| \geq \epsilon$, we can estimate

$$
\begin{aligned}
\left\|\frac{1}{\kappa+s} u\right\|^{p} & =\int_{[0, \infty)} \frac{(\kappa+1)^{p-1}}{|\kappa+s|^{p}}|u|^{p} \nu(\mathrm{d} \kappa)=\int_{[0, \infty)} \frac{|\kappa+s|^{1-p}}{(\kappa+1)^{1-p}} \frac{1}{|\kappa+s|} \nu(\mathrm{d} \kappa)|u|^{p} \\
& \leq c(\epsilon) \int_{[0, \infty)} \frac{1}{\kappa+|s|} \nu(\mathrm{d} \kappa)|u|^{p}=c(\epsilon) \hat{a}(|s|)|u|^{p} \nu(\mathrm{d} \kappa) .
\end{aligned}
$$

Therefore

$$
\begin{aligned}
\|v\| \leq \frac{1}{|s|}\|v\|+c(\epsilon)[\hat{a}(|s|)]^{1 / p}|u| \leq & \frac{1}{|s|}\|v\|+c(\epsilon) \hat{a}(|s|)^{1 / p} \frac{1}{|s| \hat{a}(|s|)} \hat{a}(s)^{1 / q}\|v\| \\
& +c(\epsilon) \frac{1}{|s| \hat{a}(|s|)} \hat{a}(s)^{1 / p}|h| \\
= & \frac{1}{|s|}\|v\|+c(\epsilon) \frac{1}{|s|}\|v\|+c(\epsilon) \frac{1}{|s| \hat{a}(|s|)^{1 / q}}|h| .
\end{aligned}
$$

Thus we have shown that $w$, given by (3.2) is in $X$, so the solution to (3.1) exists. Moreover we have proved the first parts of the estimates (3.3). The second part follows from $|s \hat{a}(s)| \geq c|s|^{\alpha(a)}$ (see Remark 2.5(3)).

Previous lemma allows to introduce the operator $P(s)$, for every $s \in \mathbb{C}^{+}$, that defines the solution of problem (3.1). More precisely, we have

Definition 3.6. For $s \in \mathbb{C}^{+}$we define the operator $P(s): E \rightarrow X$ by $P(s)(h):=$ $w$, where $w$ solves $(3.1)$ with $v=0$.

By default we write $P:=P(1)$.

Now we are ready to define the generator $B$ of our semigroup in $X$ :

Definition 3.7. Let Hypotheses 2.4 and 2.1 hold. We define the operator $B$ : $D(B) \subset X \rightarrow X$ by

$$
\begin{aligned}
D(B) & :=\left\{w \in D(\tilde{B}) \mid \tilde{J} w \in D(A), \int_{[0, \infty)}[\tilde{B} w](\kappa) \nu(\mathrm{d} \kappa)=A \tilde{J} w\right\}, \\
B w & :=\tilde{B} w .
\end{aligned}
$$

As usual, $R(s, B)$ will denote the resolvent $(s-B)^{-1}$

At the core of our state space setting is the following generation result.

Theorem 3.8. Let Hypotheses 2.4 and 2.1 hold. Then the operator $B$ is the infinitesimal generator of an analytic semigroup $e^{t B}$ on $X$. Moreover, for all $\epsilon>0$, there exists some constant $c(\epsilon)$ such that $\left\|e^{t B}\right\| \leq c(\epsilon) e^{\epsilon t}$. 
Proof. For $s \in \mathbb{C}^{+}$, the vector $R(s, B) v$ is the solution of (3.1) with $h=0$. By Lemma 3.5, $R(s, B)$ exists and $\|R(s, B)\| \leq c(\epsilon)|s|^{-1}$ if $|s| \geq \epsilon$. This implies that $B$ is sectorial with angle $\omega_{B}<\frac{\pi}{2}$, and by [19, Proposition 2.1.1.h] the operator $B$ is densely defined. Now $B$ is the generator of an analytic semigroup, whose exponential growth rate is not greater than $\epsilon$.

Under some additional condition it is possible to prove exponential stability of the semigroup $e^{t B}$. This is especially needed in the study of asymptotic results for the stochastically perturbed equation.

Proposition 3.9. Assume that for some $\sigma>0$ the function $e^{\sigma t} a(t)$ is completely monotonic.

Then the real parts of the spectrum of $B$ are bounded by some $\epsilon<0$. Consequently, the semigroup $e^{t B}$ decays exponentially.

Proof. All we need to show is that 0 is in the resolvent set of $B$. Once this is proved, the spectral bound is negative since the spectrum is confined to a sector. From this exponential decay follows, since analytic semigroups have spectrum determined growth.

Notice that $\hat{a}(s)$ exists in the set $\mathbb{C} \backslash(-\infty,-\sigma]$, and by assumption, 0 is in the resolvent set of $A$. Since from Lemma 3.5 we obtain that

$$
[R(s, B) v](\kappa)=\frac{1}{s+\kappa}\left(v(\kappa)+R(s \hat{a}(s), A)\left[\int_{[0, \infty)} \frac{\kappa}{s+\kappa} v(\kappa) \nu(\mathrm{d} \kappa)\right]\right)
$$

compare (3.2), it is easily seen that $R(s, B)$ can be analytically extended to a neighborhood of 0 .

Finally we define the operator $Q$ relating the initial function $u_{0}$ to the initial state:

Definition 3.10. We assume that Hypotheses 2.1 and 2.4 hold. We define a linear operator

$$
Q: \mathbf{L}^{1}((-\infty, 0] ; E) \rightarrow \mathbf{L}^{\infty}((-\infty, 0] ; E ; \mathrm{d} \nu)
$$

by setting

$$
Q u_{0}=\int_{0}^{\infty} e^{-\kappa \tau} u_{0}(-\tau) \mathrm{d} \tau .
$$

Notice that additional conditions are required to have $Q u_{0} \in X$. These will be discussed in Subsection 3.3.

\subsection{Regularity of $\boldsymbol{P}$ and $\boldsymbol{J}$}

Lemma 3.11. Let Hypotheses 2.1 and 2.4 hold. Let $s, t \in \mathbb{C}^{+}$. Then

$$
\begin{aligned}
& P(s)-P(t)=(t-s) R(s, B) P(t), \\
& (t-B) R(s, B) P(t)=P(s) .
\end{aligned}
$$

For $|s|>\epsilon$,

$$
\|P(s)\| \leq \frac{c(\epsilon)}{|s| \hat{a}(|s|)^{1 / q}}
$$


Moreover, if $h \in E_{-\sigma, p}$ for some $\sigma \in(0,1)$, then

$$
\|P(s) h\| \leq \frac{c(\epsilon)}{|s|^{1-\sigma} \hat{a}(|s|)^{\frac{1}{q}-\sigma}}|h|_{-\sigma, p} .
$$

Proof. Using the definition of $P$ we have for $h \in E$ :

$$
\begin{aligned}
(s-\tilde{B})[P(s)-P(t)] h & =(s-\tilde{B}) P(s) h-(t-\tilde{B}) P(t) h+(t-s) P(t) h \\
& =0+0+(t-s) P(t) h .
\end{aligned}
$$

Moreover,

$$
\begin{aligned}
\int_{[0, \infty)}[\tilde{B}(P(t)-P(s)) h](\kappa) \nu(\mathrm{d} \kappa)= & \int_{[0, \infty)}[\tilde{B} P(t) h](\kappa) \nu(\mathrm{d} \kappa) \\
& -\int_{[0, \infty)}[\tilde{B} P(s) h](\kappa) \nu(\mathrm{d} \kappa) \\
& =A \tilde{J} P(t) h+h-A \tilde{J} P(s) h-h \\
& =A \tilde{J}(P(t)-P(s)) h .
\end{aligned}
$$

Consequently,

$$
[P(t)-P(s)] h \in D(B)
$$

and

$$
(s-B)[P(s)-P(t)] h=(t-s) P(t) h .
$$

This proves (3.4). Now we obtain (3.5):

$$
\begin{aligned}
(t-B) R(s, B) P(t) & =(s-B) R(s, B) P(t)+(t-s) R(s, B) P(t) \\
& =P(t)+P(s)-P(t) .
\end{aligned}
$$

Equation (3.6) is a consequence of (3.3) for the special case $v=0$. Equation (3.7) is proved similarly, using the fact that

$$
|R(s \hat{a}(s), A) h| \leq \frac{c(\epsilon)}{(s \hat{a}(s))^{1-\sigma}}|h|_{-\sigma, p} .
$$

Lemma 3.12. Suppose that Hypotheses 2.1 and 2.4 hold.

(1) Let $0<\theta<\frac{1}{p}+\frac{\alpha(a)}{q}$. Then

$$
\int_{1}^{\infty} s^{\theta p-1}\|P(s)\|^{p} \mathrm{~d} s<+\infty .
$$

Moreover, for all $t>0$, the operator $P(t)$ maps $E$ continuously into $X_{\theta, p}$.

(2) Let $\theta, \sigma \in(0,1)$ be such that $\theta+\alpha(a) \sigma<\frac{1}{p}+\frac{\alpha(a)}{q}$. Then for all $t>0$, the operator $P(t)$ maps $E_{-\sigma, p}$ continuously into $X_{\theta, p}$. 
Proof. Fix $t>0$. According to [19, Lemma 6.1.1.] (see also [21]) we can estimate $\|w\|_{\theta, p}^{p}$ by $\int_{1}^{\infty}\left\|s^{\theta}(t-B) R(s, B) w\right\|^{p} \frac{\mathrm{d} s}{s}$. We utilize (3.5) and (3.6):

$$
\begin{aligned}
\int_{1}^{\infty} s^{\theta p-1}\|(t-B) R(s, B) P(t) h\|^{p} \mathrm{~d} s & =\int_{1}^{\infty} s^{\theta p-1}\|P(s) h\|^{p} \mathrm{~d} s \\
& \leq c \int_{1}^{\infty} s^{\theta p-1} s^{-p} \hat{a}(s)^{-p / q} \mathrm{~d} s \\
& =c \int_{1}^{\infty} s^{\theta p-p-1} \hat{a}(s)^{-p+1} \mathrm{~d} s \\
& \leq c \int_{1}^{\infty} s^{\theta p-p-1+(\alpha(a)-1)(1-p)} \mathrm{d} s .
\end{aligned}
$$

The latter integral is finite since

$$
\begin{aligned}
\theta p-p+(\alpha(a)-1)(1-p)-1 & =\theta p-\alpha(a)(p-1)-1-1 \\
& =p\left(\theta-\frac{\alpha(a)}{q}-\frac{1}{p}\right)-1<-1 .
\end{aligned}
$$

This proves (1). Assertion(2) can be proved similarly using (3.7).

Lemma 3.13. Suppose that Hypotheses 2.1 and 2.4 hold, and that $\eta \in(0,1)$ is such that:

$$
\int_{1}^{\infty} s^{q-1-q \eta}\|\tilde{J} R(s, B)\|_{\mathcal{L}(X ; E)}^{q} \mathrm{~d} s<+\infty .
$$

Then $\tilde{J}$ admits a continuous extension $J: X_{\eta, p} \rightarrow E$.

Proof. We show that for some constant $c$ independent of $s$ and some fixed $t>0$ we have, for all $w \in D(B)$ :

$$
|(s-t) \tilde{J} R(s, B) w| \leq c\|w\|_{\eta, p} .
$$

The rest follows by standard arguments. Notice that

$$
\frac{d}{d s}(s-t) \tilde{J} R(s, B) w=\tilde{J}\left[R(s, B)-(s-t) R(s, B)^{2}\right] w=\tilde{J}(t-B) R(s, B)^{2} w .
$$

Therefore

$$
\begin{aligned}
|(s-t) \tilde{J} R(s, B) w| \leq & \int_{t}^{s}|\tilde{J} R(s, B)(t-B) R(s, B) w| \mathrm{d} s \\
\leq & \int_{t}^{s} s^{1-\eta-(1 / q)}\|\tilde{J} R(s, B)\|_{\mathcal{L}(X ; E)} s^{\eta-(1 / p)} \\
& \times\|(t-B) R(s, B) w\| \mathrm{d} s \\
\leq & c\left[\int_{t}^{s} s^{q-1-q \eta}\|\tilde{J} R(s, B)\|_{\mathcal{L}(X ; E)}^{q} d s\right]^{1 / q} \\
& \times\left[\int_{t}^{s} s^{p \eta-1}\|(t-B) R(s, B) w\|^{p} d s\right]^{1 / p} \\
\leq & c\|w\|_{\eta, p} .
\end{aligned}
$$


Lemma 3.14. Suppose that Hypotheses 2.1 and 2.4 hold.

(1) Let $\frac{1}{p}-\frac{\alpha(a)}{p}<\eta<1$. Then $\tilde{J}$ admits a continuous extension $J: X_{\eta, p} \rightarrow \stackrel{p}{E}$.

(2) Let $\mu, \eta \in(0,1)$ be such that $\frac{1}{p}-\frac{\alpha(a)}{p}<\eta-\alpha(a) \mu$. Then $\tilde{J}$ admits a continuous extension $J: X_{\eta, p} \rightarrow E_{\mu, p}$.

Proof. By (3.3), applied to the case $h=0$, we have for $s>\epsilon$

$$
|\tilde{J} R(s, B) w| \leq c(\epsilon) s^{-1} \hat{a}(s)^{(1 / q)-1}\|w\| .
$$

Thus

$$
\begin{aligned}
\int_{1}^{\infty} s^{q-1-q \eta}\|\tilde{J} R(s, B)\|_{\mathcal{L}(X ; E)}^{q} \mathrm{~d} s & \leq c \int_{1}^{\infty} s^{-1-q \eta} \hat{a}(s)^{1-q} \mathrm{~d} s \\
& \leq c \int_{1}^{\infty} s^{-1-q \eta-(\alpha(a)-1)(1-q)} \mathrm{d} s
\end{aligned}
$$

The latter integral is finite, since

$$
-q \eta+(1-\alpha(a))(q-1)-1=q\left[-\eta+\frac{1}{p}(1-\alpha(a))\right]-1<-1 .
$$

Therefore (3.9) holds and Lemma 3.13 yields Assertion(1). Assertion(2) is proved similarly, using the estimate

$|\tilde{J} R(s, B) w|_{\mu, p}=\left|R(s \hat{a}(s), A) \int_{[0, \infty)} \frac{\kappa}{\kappa+s} w(\kappa) \nu(\mathrm{d} \kappa)\right|_{\mu, p} \leq c(s \hat{a}(s))^{\mu-1} \hat{a}(s)^{1 / q}$.

\subsection{Regularity of $Q$}

In this subsection we will give sufficient conditions on $u_{0}$ to control the smoothness of $Q u_{0}$. We begin with some useful estimates.

Lemma 3.15. Assume that Hypotheses 2.1 and 2.4 hold. Let $\zeta \in(0, p), \delta \in$ $[0,2), \rho \in[0,1]$ and $\left.u_{0}:(-\infty, 0] \rightarrow E\right)$ be measurable such that

$$
\begin{aligned}
& \int_{0}^{\infty}\left|u_{0}(-\tau)\right| \mathrm{d} \tau<+\infty \\
& \int_{0}^{\infty} a(\tau)\left|u_{0}(-\tau)\right| \mathrm{d} \tau<+\infty \\
& \int_{0}^{\infty} \tau^{-\delta}\left|u_{0}(-\tau)\right| \mathrm{d} \tau<+\infty \\
& \int_{0}^{\infty}[a(\tau)-a(\tau+\epsilon)]\left|u_{0}(-\tau)\right| \mathrm{d} \tau<\epsilon^{\rho} .
\end{aligned}
$$

For $\kappa \in[0, \infty)$ and $t \geq 1$ put

$$
\begin{aligned}
v_{0}(\kappa) & :=\int_{0}^{\infty} e^{-\kappa \tau} u_{0}(-\tau) \mathrm{d} \tau, \\
w(s) & :=\int_{[0, \infty)} \frac{\tilde{\kappa}}{s+\tilde{\kappa}} v_{0}(\tilde{\kappa}) \nu(\mathrm{d} \tilde{\kappa}),
\end{aligned}
$$

i.e., $v_{0}=Q u_{0}$ and $R(s \hat{a}(s), A) w(s)=J R(s, B) v_{0}$. 
Then the following estimates hold (with constants $M$ independent of $s, \kappa$ ):

$$
\begin{aligned}
& \left|v_{0}(\kappa)\right| \leq M(\kappa+1)^{-\delta}, \\
& \int_{[0, \infty)}\left|v_{0}(\kappa)\right| \nu(\mathrm{d} \kappa) \leq M, \\
& |w(s)| \leq M, \\
& |w(s)| \leq M s^{1-\delta} \quad \text { if } \delta>1, \\
& |w(s)| \leq M s^{-\rho}, \\
& \int_{1}^{\infty} \frac{s^{\zeta-1}}{(\kappa+s)^{p}} d s \leq M(\kappa+1)^{\zeta-p} .
\end{aligned}
$$

Proof. We start with (3.10)

$$
\begin{aligned}
\int_{0}^{\infty} e^{-\kappa \tau}\left|u_{0}(-\tau)\right| \mathrm{d} \tau & =\int_{0}^{\infty} e^{-\kappa \tau} \tau^{\delta} \tau^{-\delta}\left|u_{0}(-\tau)\right| \mathrm{d} \tau \\
& \leq \kappa^{-\delta} \max _{\tau \geq 0}\left(e^{-\kappa \tau}(\kappa \tau)^{\delta}\right) \int_{0}^{\infty} \tau^{-\delta}\left|u_{0}(-\tau)\right| \mathrm{d} \tau \leq M \kappa^{-\delta} .
\end{aligned}
$$

The same estimate with $\delta=0$ yields $\left|v_{0}(\kappa)\right| \leq M$, so that finally $\left|v_{0}(\kappa)\right| \leq$ $M(\kappa+1)^{-\delta}$. This proves (3.10). The proof of (3.11) is a simple application of Fubini:

$$
\begin{aligned}
\int_{[0, \infty)}\left|v_{0}(\kappa)\right| \nu(\mathrm{d} \kappa) & \leq \int_{[0, \infty)} \int_{0}^{\infty} e^{-\kappa \tau}\left|u_{0}(-\tau)\right| d \tau \nu(\mathrm{d} \kappa) \\
& =\int_{0}^{\infty} \int_{[0, \infty)} e^{-\kappa \tau}\left|u_{0}(-\tau)\right| \nu(\mathrm{d} \kappa) \mathrm{d} \tau=\int_{0}^{\infty} a(\tau)\left|u_{0}(-\tau)\right| \mathrm{d} \tau
\end{aligned}
$$

The estimate (3.12) follows easily from (3.11):

$$
\int_{[0, \infty)} \frac{\kappa}{\kappa+s}\left|v_{0}(\kappa)\right| \nu(\mathrm{d} \kappa) \leq \int_{[0, \infty)}\left|v_{0}(\kappa)\right| \nu(\mathrm{d} \kappa) \leq M .
$$

If $\delta>1$ we use (3.10) and estimate

$$
\begin{aligned}
\int_{[0, \infty)} \frac{\kappa}{\kappa+s}\left|v_{0}(\kappa)\right| \nu(\mathrm{d} \kappa) & \leq M \int_{[0, \infty)} \frac{\kappa}{\kappa+s}(\kappa+1)^{-\delta} \nu(\mathrm{d} \kappa) \\
& \leq M \int_{[0, \infty)} \frac{(\kappa+1)^{2-\delta}}{(\kappa+s)^{2-\delta}} \frac{1}{(\kappa+s)^{\delta-1}} \frac{1}{\kappa+1} \nu(\mathrm{d} \kappa) \\
& \leq M s^{1-\delta} \int_{[0, \infty)} \frac{1}{\kappa+1} \nu(\mathrm{d} \kappa)=M \hat{a}(1) s^{1-\delta} .
\end{aligned}
$$

This proves (3.13). 
To prove (3.14) notice that

$$
\begin{aligned}
|w(s)| \leq & \int_{[0, \infty)} \frac{\kappa}{\kappa+s} \int_{0}^{\infty} e^{-\kappa \tau}\left|u_{0}(-\tau)\right| \mathrm{d} \tau \nu(\mathrm{d} \kappa) \\
= & \int_{[0, \infty)} \int_{0}^{\infty}\left(1-s \int_{0}^{\infty} e^{-(\kappa+s) \sigma} \mathrm{d} \sigma\right) e^{-\kappa \tau}\left|u_{0}(-\tau)\right| \mathrm{d} \tau \nu(\mathrm{d} \kappa) \\
= & \int_{0}^{\infty} \int_{[0, \infty)} e^{-\kappa \tau} \nu(\mathrm{d} \kappa)\left|u_{0}(-\tau)\right| \mathrm{d} \tau \\
& -\int_{0}^{\infty} \int_{0}^{\infty} s e^{-s \sigma}\left(\int_{[0, \infty)} e^{-\kappa(\sigma+\tau)} \nu(\mathrm{d} \kappa)\right)\left|u_{0}(-\tau)\right| \mathrm{d} \tau \\
= & \int_{0}^{\infty} a(\tau)\left|u_{0}(-\tau)\right| \mathrm{d} \tau+\int_{0}^{\infty} s e^{-\sigma s} \int_{0}^{\infty} a(\tau+\sigma)\left|u_{0}(-\tau)\right| \mathrm{d} \tau \mathrm{d} \sigma \\
= & \int_{0}^{\infty} s e^{-\sigma s} \int_{0}^{\infty}[a(\tau)-a(\tau+\sigma)]\left|u_{0}(-\tau)\right| \mathrm{d} \tau \mathrm{d} \sigma \\
\leq & M \int_{0}^{\infty} \sigma^{\rho} e^{-\sigma s} s \mathrm{~d} \sigma=M \int_{0}^{\infty} s^{-\rho} \gamma^{\rho} e^{-\gamma} \mathrm{d} \gamma=M s^{-\rho} .
\end{aligned}
$$

Finally we prove (3.15):

$$
\int_{1}^{\infty} \frac{s^{\zeta-1}}{(\kappa+s)^{p}} \mathrm{~d} t=\int_{1 / \kappa}^{\infty} \kappa^{\zeta-p} \frac{\tau^{\zeta-1}}{(1+\tau)^{p}} \mathrm{~d} \tau \leq \kappa^{\zeta-p} \int_{0}^{\infty} \frac{\tau^{\zeta-1}}{(1+\tau)^{p}} \mathrm{~d} \tau \leq M \kappa^{\zeta-p} .
$$

On the other hand

$$
\int_{1}^{\infty} \frac{s^{\zeta-1}}{(\kappa+s)^{p}} \mathrm{~d} s \leq \int_{1}^{\infty} s^{\zeta-p-1} \mathrm{~d} s<+\infty
$$

These two estimates imply (3.15).

Lemma 3.16. Assume that Hypotheses 2.1 and 2.4 hold. Let $u_{0}:(-\infty, 0) \rightarrow E$ be a measurable function and put $v_{0}=Q u_{0}$. Then the following assertions hold:

(1) Suppose that $u_{0} \in \mathbf{L}^{1}((-\infty, 0) ; E) \cap \mathbf{L}^{\infty}((-\infty, 0) ; E)$. Then $v_{0} \in X$.

(2) Suppose that $\rho \in[0,1], \delta \in[0,1), \chi \in(0,1)$ are such that

$$
\begin{aligned}
& \chi-1 \leq \rho-\frac{1-\alpha(a)}{q}, \quad \chi-1 \leq \frac{\delta-1}{q} \\
& \int_{0}^{\infty}\left|u_{0}(-\tau)\right| \mathrm{d} \tau<+\infty \\
& \int_{0}^{\infty} \tau^{-\delta}\left|u_{0}(-\tau)\right| \mathrm{d} \tau<+\infty \\
& \int_{0}^{\infty} a(\tau)\left|u_{0}(-\tau)\right| \mathrm{d} \tau<+\infty, \\
& \int_{0}^{\infty}[a(\tau)-a(\epsilon+\tau)]\left|u_{0}(-\tau)\right| \mathrm{d} \tau \leq M \epsilon^{\rho} \quad \text { for all } \epsilon>0 .
\end{aligned}
$$

Then $v_{0} \in X_{\chi-1, p}$. 
(3) Suppose that $\mu \in[1 / p, 1), \delta \in(1,2), \chi \in(0,1)$, and $u_{0}(0) \in E_{\mu, p}$ are such that

$$
\begin{aligned}
& \chi<\alpha(a) \mu+\frac{1-\alpha(a)}{p}, \quad \chi<\frac{\delta-1}{q}, \quad \chi<(\delta-1)-\frac{1-\alpha(a)}{q}, \\
& \int_{0}^{\infty}\left|u_{0}(-\tau)\right| \mathrm{d} \tau<+\infty \\
& \int_{0}^{\infty} \tau^{-\delta}\left|u_{0}(-\tau)-u_{0}(0)\right| \mathrm{d} \tau<+\infty \\
& \int_{0}^{\infty} a(\tau)\left|u_{0}(-\tau)\right| \mathrm{d} \tau<+\infty
\end{aligned}
$$

Then $v_{0} \in X_{\chi, p}$.

Proof. Assertion (1). Notice that $\left|v_{0}(\kappa)\right| \leq \kappa^{-1}\left\|u_{0}\right\|_{\mathbf{L}^{\infty}}$ and $\left|v_{0}(\kappa)\right| \leq\left\|u_{0}\right\|_{\mathbf{L}^{1}}$. Thus $(\kappa+1)\left|v_{0}(\kappa)\right|$ is bounded on $(-\infty, 0]$. Consequently we have the following estimate:

$$
\begin{aligned}
\left\|v_{0}\right\|_{X}^{p} & =\int_{[0, \infty)}(\kappa+1)^{p-1}\left|v_{0}(\kappa)\right|^{p} \nu(\mathrm{d} \kappa)=\int_{[0, \infty)}(\kappa+1)^{p}\left|v_{0}(\kappa)\right|^{p} \frac{1}{\kappa+1} \nu(\mathrm{d} \kappa) \\
& \leq \int_{[0, \infty)} M^{p} \frac{1}{\kappa+1} \nu(\mathrm{d} \kappa)=M^{p} \hat{a}(1)<\infty .
\end{aligned}
$$

Assertion (2). We have to show that $R(1, B) v_{0} \in X_{\eta, p}$, i.e.,

$$
\int_{1}^{\infty} s^{p \chi-1}\left\|R(s, B) v_{0}\right\|^{p} \mathrm{~d} s<+\infty .
$$

Now,

$$
\begin{aligned}
{\left[R(s, B) v_{0}\right](\kappa) } & =\frac{1}{s+\kappa} v_{0}(\kappa)+\frac{1}{s+\kappa} R(s \hat{a}(s), A) w(s) \\
& =\frac{1}{s+\kappa} v_{0}(\kappa)+[P(s) w(s)](\kappa) \quad \text { with } \\
w(s) & =\int_{[0, \infty)} \frac{\kappa}{\kappa+s} v_{0}(\kappa) \nu(\mathrm{d} \kappa) .
\end{aligned}
$$

We estimate the two parts separately. As far as the first one is concerned,

$$
\begin{aligned}
\int_{1}^{\infty} s^{p \chi-1}\left\|\frac{1}{s+\kappa} v_{0}\right\|^{p} \mathrm{~d} s= & \int_{1}^{\infty} \int_{[0, \infty)}(\kappa+1)^{p-1} \\
& \times \frac{s^{p \chi-1}}{(s+\kappa)^{p}}\left|v_{0}(\kappa)\right|^{p-1}\left|v_{0}(\kappa)\right| \nu(\mathrm{d} \kappa) \mathrm{d} s
\end{aligned}
$$

then use (3.10):

$$
\leq M \int_{[0, \infty)}\left(\int_{1}^{\infty} \frac{s^{p \chi-1}}{(s+\kappa)^{p}} \mathrm{~d} s\right)(\kappa+1)^{p-1-\delta(p-1)}\left|v_{0}(\kappa)\right| \nu(\mathrm{d} \kappa)
$$

use (3.15):

$$
\leq M \int_{[0, \infty)}(\kappa+1)^{p \chi-p+p-1-(p-1) \delta}\left|v_{0}(\kappa)\right| \nu(\mathrm{d} \kappa)
$$


use $p \chi-1-(p-1) \delta=p\left(\chi-\frac{1}{p}-\frac{\delta}{q}\right) \leq 0$ :

$$
\leq M \int_{[0, \infty)}\left|v_{0}(\kappa)\right| \nu(\mathrm{d} \kappa)
$$

and finally by (3.11):

$$
\int_{1}^{\infty} s^{p \chi-1}\left\|\frac{1}{s+\kappa} v_{0}\right\|^{p} \mathrm{~d} s<+\infty .
$$

For the second part we use (3.14) and Lemma 3.12 with $\theta$ replaced by $\chi-\rho$ :

$$
\int_{1}^{\infty} s^{p \chi-1}\|P(s) w(s)\|^{p} \mathrm{~d} s \leq M \int_{1}^{\infty} s^{p \chi-p \rho-1}\|P(s)\|^{p} \mathrm{~d} s<+\infty .
$$
with

Assertion (3). We put $h=u_{0}(0)$ and split $u_{0}$ in two parts $u_{0}=u_{1}+u_{2}$

$$
u_{0}=e^{\tau} h+\left(u_{0}(\tau)-e^{\tau} h\right)=: u_{1}(\tau)+u_{2}(\tau) .
$$

We will estimate the two functions $v_{1}=Q u_{1}$ and $v_{2}=Q u_{2}$ separately. In any case we have to show that

$$
\int_{1}^{\infty} s^{p \chi-1}\left\|(1-B) R(s, B) v_{i}\right\|^{p} \mathrm{~d} s<+\infty .
$$

Now

$$
\begin{aligned}
{\left[(1-B) R(s, B) v_{i}\right](\kappa) } & =\left[v_{i}-(s-1) R(s, B) v_{i}\right](\kappa) \\
& =\frac{\kappa+1}{\kappa+s} v_{i}(\kappa)-\frac{s-1}{\kappa+s} R(s \hat{a}(s), A) w_{i}(s) \quad \text { with } \\
w_{i}(s) & =\int_{[0, \infty)} \frac{\kappa}{\kappa+s} v_{i}(\kappa) \nu(\mathrm{d} \kappa) .
\end{aligned}
$$

We treat first $u_{1}(\tau):=e^{\tau} h$.

$$
\begin{aligned}
v_{1}(\kappa) & =\int_{0}^{\infty} e^{-\kappa \tau-\tau} h \mathrm{~d} \tau=\frac{1}{1+\kappa} h \\
w_{1}(s) & =\int_{[0, \infty)} \frac{\kappa}{(1+\kappa)(s+\kappa)} \nu(\mathrm{d} \kappa) h \\
& =\frac{1}{s-1} \int_{[0, \infty)}\left(\frac{s}{\kappa+s}-\frac{1}{\kappa+1}\right) \nu(\mathrm{d} \kappa) h=\frac{s \hat{a}(s)-\hat{a}(1)}{s-1} h .
\end{aligned}
$$

Thus

$$
\begin{aligned}
{\left[(1-B) R(s, B) v_{1}\right](\kappa)=} & \frac{1}{\kappa+s}\left(\frac{\kappa+1}{\kappa+1} h-s \hat{a}(s) R(s \hat{a}(s), A) h\right. \\
& +\hat{a}(1) R(s \hat{a}(s), A) h) \\
= & -\frac{1}{\kappa+s}[A+\hat{a}(1)] R(s \hat{a}(s), A) h .
\end{aligned}
$$

We use that $h \in E_{\mu, p}$ which implies that

$$
|(\hat{a}(1)+A) R(s \hat{a}(s), A) h| \leq M(s \hat{a}(s))^{-\mu} .
$$


Thus we obtain

$$
\begin{aligned}
& \int_{1}^{\infty} s^{p \chi-1}\left|\frac{1}{\kappa+s}(\hat{a}(1)+A) R(s \hat{a}(s), A) h\right|^{p} \mathrm{~d} s \\
& \quad \leq \int_{1}^{\infty} \int_{[0, \infty)} s^{p \chi-1} \frac{(\kappa+1)^{p-1}}{(\kappa+s)^{p}}(s \hat{a}(s))^{-\mu p} \nu(\mathrm{d} \kappa) \mathrm{d} s \\
& \quad \leq \int_{1}^{\infty} \int_{[0, \infty)} s^{p \chi-1}(s \hat{a}(s))^{-p \mu} \frac{1}{\kappa+s} \nu(\mathrm{d} \kappa) \mathrm{d} s \leq \int_{1}^{\infty} s^{p \chi-1}(s \hat{a}(s))^{-p \mu} \hat{a}(s) \mathrm{d} s \\
& \quad=\int_{1}^{\infty} s^{p \chi-2}(s \hat{a}(s))^{1-p \mu} \mathrm{d} s
\end{aligned}
$$

here we use $p \mu-1 \geq 0$ and $s \hat{a}(s) \geq M s^{\alpha(a)}$ :

$\int_{1}^{\infty} s^{p \chi-1}\left|\frac{1}{\kappa+s}(\hat{a}(1)+A) R(s \hat{a}(s), A) h\right|^{p} \mathrm{~d} s \leq \int_{1}^{\infty} s^{p \chi-2+\alpha(a)(1-p \mu)} \mathrm{d} s<+\infty$ since we can estimate

$$
p \chi-2+\alpha(a)(1-p \mu)=p\left(\chi-\alpha(a) \mu-\frac{1-\alpha(a)}{p}\right)-1<-1 .
$$

We estimate now $v_{2}=Q u_{2}$. Notice that $u_{2}$ satisfies the same conditions as $u_{0}$, with $h$ replaced by 0 . Again we have to estimate two parts:

$$
\begin{aligned}
{\left[(1-B) R(s, B) v_{2}\right](\kappa) } & =\frac{\kappa+1}{\kappa+s} v_{2}(\kappa)-\frac{s-1}{\kappa+s} R(s \hat{a}(s), A) w_{2}(s) \\
& =\frac{\kappa+1}{\kappa+s} v_{2}(\kappa)-(s-1)\left[P(s) w_{2}(s)\right](\kappa) .
\end{aligned}
$$

Now

$$
\begin{aligned}
\int_{1}^{\infty} s^{p \chi-1}\left\|\frac{\kappa+1}{\kappa+s} v_{2}\right\|^{p} \mathrm{~d} s= & \int_{1}^{\infty} \int_{[0, \infty)} s^{p \chi-1} \frac{(\kappa+1)^{2 p-1}}{(\kappa+s)^{p}}\left|v_{2}(\kappa)\right|^{p-1} \\
& \times\left|v_{2}(\kappa)\right| \nu(\mathrm{d} \kappa) \mathrm{d} s
\end{aligned}
$$

use (3.10):

$$
\leq M \int_{[0, \infty)}\left(\int_{1}^{\infty} \frac{s^{p \chi-1}}{(\kappa+s)^{p}} d s\right)(\kappa+1)^{2 p-1-\delta(p-1)}\left|v_{2}(\kappa)\right| \nu(\mathrm{d} \kappa)
$$

use (3.15):

$$
\begin{aligned}
& \leq M \int_{[0, \infty)}(\kappa+1)^{p \chi+p-1-\delta(p-1)}\left|v_{2}(\kappa)\right| \nu(\mathrm{d} \kappa) \\
& \text { use } p \chi+(p-1)(1-\delta)=p\left(\chi-\frac{\delta-1}{q}\right) \leq 0 \text { and }(3.11) \text { : } \\
& \quad \leq M \int_{[0, \infty)}\left|v_{2}(\kappa)\right| \nu(\mathrm{d} \kappa)<+\infty .
\end{aligned}
$$


On the other hand, by (3.13):

$$
\begin{aligned}
\int_{1}^{\infty} s^{p \chi-1}\|(s-1) P(s) w(s)\|^{p} \mathrm{~d} s & \leq \int_{1}^{\infty} s^{p \chi+p-p(\delta-1)-1}\|P(s)\|^{p} \mathrm{~d} s \\
& =\int_{1}^{\infty} s^{p(\chi+1-(\delta-1))-1}\|P(s)\|^{p} \mathrm{~d} s<+\infty .
\end{aligned}
$$

In the last step we have used Lemma 3.12 with $\theta$ replaced by $\chi-(\delta-1)+1$ and the fact that

$$
\chi-(\delta-1)+1<\frac{1}{p}+\frac{\alpha(a)}{q} .
$$

\section{Application to deterministic integral equation}

\subsection{Proof of Theorem 2.7}

The proof will be given by Laplace transforms.

Lemma 4.1. We assume that Hypotheses 2.1 and 2.4 hold. Let $u_{0} \in$ $\mathbf{L}^{1}((-\infty, 0] ; E)$ with

$$
\int_{0}^{\infty} a(\tau)\left|u_{0}(-\tau)\right| \mathrm{d} \tau<+\infty
$$

let $f:[0, \infty) \rightarrow E$ and $u_{+}:[0, \infty) \rightarrow E$ be exponentially integrable functions. Put $v_{0}=Q u_{0}$ and

$$
u(t)= \begin{cases}u_{0}(t) & \text { if } t \leq 0 \\ u_{+}(t) & \text { if } t>0\end{cases}
$$

Then $u$ is an integrated solution of (1.1) if and only if the following equation holds for sufficiently large $s \in \mathbb{R}$ :

$$
(s \hat{a}(s)-A) \hat{u}_{+}(s)=\int_{[0, \infty)} \frac{\kappa}{\kappa+s} v_{0}(\kappa) \nu(\mathrm{d} \kappa)+\hat{f}(s) .
$$

Proof. Since $a(\tau) u_{0}(\tau)$ is integrable on $(-\infty, 0]$ and $a(t)$ is decreasing, the function $t \mapsto \int_{-\infty}^{0} a(t-\tau) u_{0}(\tau) \mathrm{d} \tau$ is bounded. Next we compute

$$
\begin{aligned}
\int_{-\infty}^{0}(a(t-\tau)-a(-\tau)) u_{0}(\tau) \mathrm{d} \tau & =\int_{-\infty}^{0} \int_{[0, \infty)}\left(e^{-\kappa(t-\tau)}-e^{\kappa \tau}\right) u_{0}(\tau) \nu(\mathrm{d} \kappa) \mathrm{d} \tau \\
& =\int_{[0, \infty)}\left(e^{-\kappa t}-1\right) \int_{0}^{\infty} e^{\kappa \tau} u_{0}(\tau) \mathrm{d} \tau \nu(\mathrm{d} \kappa) \\
& =\int_{[0, \infty)}\left(e^{-\kappa t}-1\right) v_{0}(\kappa) \nu(\mathrm{d} \kappa) .
\end{aligned}
$$


We take Laplace transforms and obtain for all $s>0$

$$
\begin{aligned}
\mathcal{L}\left[\int_{-\infty}^{0}(a(t-\tau)-a(-\tau)) u_{0}(\tau) \mathrm{d} \tau\right](s) & =\int_{[0, \infty)}\left(\frac{1}{\kappa+s}-\frac{1}{s}\right) v_{0}(\kappa) \nu(\mathrm{d} \kappa) \\
& =-\frac{1}{s} \int_{[0, \infty)} \frac{\kappa}{\kappa+s} v_{0}(\kappa) \nu(\mathrm{d} \kappa) .
\end{aligned}
$$

Now

$$
\begin{aligned}
& \int_{\infty}^{t} a(t-\tau) u(\tau) \mathrm{d} \tau-\int_{-\infty}^{t} a(-\tau) u(\tau) \mathrm{d} \tau=A \int_{0}^{t} u_{+}(\tau) \mathrm{d} \tau+\int_{0}^{t} f(\tau) \mathrm{d} \tau \\
& \Longleftrightarrow(1-A) \int_{0}^{t} u_{+}(\tau) \mathrm{d} \tau=\int_{0}^{t} u_{+}(\tau) \mathrm{d} \tau-\int_{-\infty}^{0}(a(t-\tau)-a(-\tau)) u_{0}(\tau) \mathrm{d} \tau \\
& -\int_{0}^{t} a(t-\tau) u_{+}(\tau) \mathrm{d} \tau+\int_{0}^{t} f(\tau) \mathrm{d} \tau \\
& \Longleftrightarrow \int_{0}^{t} u_{+}(\tau) \mathrm{d} \tau=R(1, A)\left[\int_{0}^{t} u_{+}(\tau) \mathrm{d} \tau-\int_{-\infty}^{0}(a(t-\tau)-a(-\tau)) u_{0}(\tau) \mathrm{d} \tau\right. \\
& \left.-\int_{0}^{t} a(t-\tau) u_{+}(\tau) \mathrm{d} \tau+\int_{0}^{t} f(\tau) \mathrm{d} \tau\right] \\
& \Longleftrightarrow \frac{1}{s} \hat{u}_{+}(s)=R(1, A)\left[\frac{1}{s} \hat{u}_{+}(s)+\frac{1}{s} \int_{[0, \infty)} \frac{\kappa}{s+\kappa} v_{0}(\kappa) \nu(\mathrm{d} \kappa)\right. \\
& \left.-\hat{a}(s) \hat{u}_{+}(s)+\frac{1}{s} \hat{f}(s)\right] \\
& \Longleftrightarrow \hat{u}_{+}(s)-A \hat{u}_{+}(s)=\hat{u}_{+}(s)+\int_{[0, \infty)} \frac{\kappa}{s+\kappa} v_{0}(\kappa) \nu(\mathrm{d} \kappa)-s \hat{a}(s) \hat{u}_{+}(s)+\hat{f}(s) \\
& \Longleftrightarrow s \hat{a}(s) \hat{u}_{+}(s)-A \hat{u}_{+}(s)=\int_{[0, \infty)} \frac{\kappa}{s+\kappa} v_{0}(\kappa) \nu(\mathrm{d} \kappa)+\hat{f}(s) .
\end{aligned}
$$

Proof of Theorem 2.7. Assertion (1). This follows from (2.6) and Lemma 3.16(2) (with $\delta=\rho=0, \chi=\frac{1}{p}$ ), Lemma 3.12, and Lemma 3.14.

Assertion (2). Since $e^{t B}$ is an analytic semigroup, and $\theta>\eta$ and $\frac{1}{p}>\eta$, for each $t>0$ the operator $e^{t B}$ maps $X_{-1 / p, p}$ into $X_{\eta, p}$ and $(1-B) e^{t B}$ maps $X_{\theta, p}$ into $X_{\eta, p}$. Moreover, for each compact interval $[0, T]$ there exists a constant $M$ such that for $t \in[0, T]$ :

$\left\|e^{t B}\right\|_{\mathcal{L}\left(X_{-1 / q, p}, X_{\eta, p}\right)} \leq M t^{-\eta-1 / q}, \quad$ and $\quad\left\|(1-B) e^{t B}\right\|_{\mathcal{L}\left(X_{\theta, p}, X_{\eta, p}\right)} \leq M t^{\theta-\eta-1}$.

Hence $\left\|e^{t B} v_{0}\right\|_{\eta, p} \leq M t^{-\eta-1 / q}$ and since $-\eta-\frac{1}{q}>-\frac{1}{p}-\frac{1}{q}=-1$ we conclude $e^{t B} v_{0} \in \mathbf{L}^{1}\left((0, T) ; X_{\eta, p}\right)$. Similarly,

$$
\left\|\int_{0}^{t}(1-B) e^{(t-s) B} \operatorname{Pf}(s) \mathrm{d} s\right\|_{\eta, p} \leq M \int_{0}^{t}(t-s)^{\theta-\eta-1}|f(s)| \mathrm{d} s .
$$


Since $t^{\theta-\eta-1}$ and $f(t)$ are integrable on finite intervals, the convolution $t^{\theta-\eta-1} *$ $|f(t)|$ is in $\mathbf{L}^{1}((0, T) ; \mathbb{R})$.

Assertion (3). We compute the Laplace transform of $\left.u\right|_{[0, \infty)}=J v$. We use standard semigroup theory and subsequently Lemmas 3.5 and 3.11:

$$
\begin{aligned}
\hat{J} v(s) & =J R(s, B) v_{0}+J(1-B) R(s, B) P(1) \hat{f}(s) \\
& =R(s \hat{a}(s), A) \int_{[0, \infty)} \frac{\kappa}{\kappa+s} v_{0}(\kappa) \nu(d \kappa)+R(s \hat{a}(s), A) \hat{f}(s) \\
& =R(s \hat{a}(s), A)\left(\int_{[0, \infty)} \frac{\kappa}{\kappa+s} v_{0}(\kappa) \nu(\mathrm{d} \kappa)+\hat{f}(s)\right) .
\end{aligned}
$$

Lemma 4.1 implies now that $u$ is an integrated solution of (1.1).

\subsection{Further regularity of the integrated solution}

The construction above is fairly technical and heavily relies on the machinery developed in previous sections. However, since the method involves analytic semigroups and interpolation spaces, once the method is understood, many additional regularity results can be obtained. We focus on two examples.

Corollary 4.2. Let Hypotheses 2.1 and 2.4 hold. Let $u_{0}:(-\infty, 0] \rightarrow E$ and $\delta>1$ be such that

$$
\begin{aligned}
& \int_{0}^{\infty}(1+a(\tau))\left|u_{0}(-\tau)\right| \mathrm{d} \tau<+\infty \\
& \int_{0}^{\infty} \tau^{-\delta}\left|u_{0}(-\tau)-u_{0}(0)\right| \mathrm{d} \tau<+\infty \\
& u_{0}(0) \in E_{1 / p, p}
\end{aligned}
$$

Let $f=0$. Then the integrated solution of (1.1) is continuous.

Proof. We will show that $v_{0} \in X_{\eta, p}$ for some $\eta>\frac{1}{p}-\frac{\alpha(a)}{p}$. Then $v(t)=e^{t} B v_{0}$ is continuous from $[0, \infty)$ into $X_{\eta, p}$, and according to Lemma 3.14, the function $u=J v$ is continuous from $[0, \infty)$ into $E$. Put $\mu=\frac{1}{p}$, choose $\eta \in\left(\frac{1}{p}-\frac{\alpha(a)}{p}, \frac{1}{p}\right)$. Then the conditions of Lemma 3.16, Assertion(3) are satisfied. Thus $v_{0} \in X_{\eta, p}$.

Corollary 4.3. Let Hypotheses 2.1 and 2.4 hold. Let $\mu, \sigma \in(0,1), r>1$ be such that $\alpha(a)(1-\mu+\sigma)>\frac{1}{r}$. Let $f \in \mathbf{L}^{r}\left([0, T], E_{-\sigma, p}\right)$ and $u_{0}=0$. Then the solution $u$ of (1.1) is Hölder continuous with values in $E_{\mu, p}$ with exponent $\alpha(a)(1-\mu-\sigma)-\frac{1}{r}-\epsilon$ for all $\epsilon>0$.

Proof. We can choose $\theta<\frac{1}{p}+\frac{\alpha(a)}{q}-\alpha(a) \sigma$ and $\eta>\frac{1}{p}-\frac{\alpha(a)}{q}+\alpha(a) \mu$ such that $\theta-\eta>\frac{1}{r}+\epsilon$. Let $s$ be the conjugate exponent to $r$. If $w_{0} \in X_{\theta}$ and $h>0$, then 


$$
\begin{aligned}
\left\|(1-B) e^{(t+h) B} w_{0}-(1-B) e^{t B} w_{0}\right\|_{\eta, p} & \leq \int_{t}^{t+h}\left\|B e^{\frac{\tau}{2} B}(1-B) e^{\frac{\tau}{2} B} w_{0}\right\|_{\eta, p} \mathrm{~d} \tau \\
& \leq M \int_{t}^{t+h} \tau^{\theta-\eta-2}\left\|w_{0}\right\|_{\theta, p} \mathrm{~d} \tau \\
& \leq M h\left(t+\frac{h}{2}\right)^{\theta-\eta-2}\left\|w_{0}\right\|_{\theta, p} .
\end{aligned}
$$

In the last step we used the fact that $t^{-\gamma}$ is convex for $\gamma:=\eta+2-\theta>0$. Now notice that $P f \in \mathbf{L}^{r}\left([0, T] ; X_{\theta, p}\right)$. Thus we can estimate

$$
\begin{aligned}
& \left\|\int_{0}^{t}(1-B) e^{(t+h-\tau) B} \operatorname{Pf}(\tau)-(1-B) e^{(t-\tau) B} \operatorname{Pf}(\tau) \mathrm{d} \tau\right\|_{\eta, p} \\
& \quad \leq M h \int_{0}^{t}\left(t-\tau+\frac{h}{2}\right)^{\theta-\eta-2}\|\operatorname{Pf}(\tau)\|_{\theta, p} \mathrm{~d} \tau \\
& \quad \leq M h\left\|\left(\tau+\frac{h}{2}\right)^{\theta-\eta-2}\right\|\left\|_{\mathbf{L}^{s}([0, T] ; \mathbb{R})}\right\| P f \|_{\mathbf{L}^{r}\left([0, T], X_{\theta, p}\right)} .
\end{aligned}
$$

Now

$$
\int_{h / 2}^{T+h / 2} \tau^{s(\theta-\eta-2)} \mathrm{d} \tau \leq \int_{h / 2}^{\infty} \tau^{s(\theta-\eta-2)} \mathrm{d} \tau=M h^{s(\theta-\eta-2+1 / s)} .
$$

Thus

$$
\left\|\left(\tau+\frac{h}{2}\right)^{\theta-\eta-2}\right\|_{\mathbf{L}^{s}([0, T] ; \mathbb{R})} \leq M h^{\theta-\eta-1-1 / r}
$$

Consequently we have

$$
\begin{aligned}
& \left\|\int_{0}^{t}\left[(1-B) e^{(t+h-\tau) B} \operatorname{Pf}(\tau)-(1-B) e^{(t-\tau) B} \operatorname{Pf}(\tau)\right] d \tau\right\|_{\eta, p} \\
& \quad \leq M h^{\theta-\eta-1 / r}\|f\|_{\mathbf{L}^{r}\left([0, T] ; X_{-\sigma, p}\right)} .
\end{aligned}
$$

Moreover we can estimate

$$
\begin{aligned}
\left\|\int_{t}^{t+h}(1-B) e^{(t+h-\tau) B} \operatorname{Pf}(\tau) \mathrm{d} \tau\right\|_{\eta, p} & \leq M \int_{0}^{h} \tau^{\theta-\eta-1}\|P f(\tau)\|_{\theta, p} \mathrm{~d} \tau \\
\leq & M\left[\int_{0}^{h} \tau^{(\theta-\eta-1) s} \mathrm{~d} \tau\right]^{1 / s} \\
& \times\|P f\|_{\mathbf{L}^{r}\left([0, T] ; X_{\theta, p}\right)} \\
= & M h^{\theta-\eta-1+\frac{1}{s}}\|f\|_{\mathbf{L}^{r}\left([0, T] ; X_{-\sigma, p}\right)} .
\end{aligned}
$$


Finally, since $J: X_{\eta, p} \rightarrow E_{\mu, p}$ is continuous, we have

$$
\begin{aligned}
|u(t+h)-u(t)|_{\mu, p} \leq & M \| \int_{0}^{t}\left[(1-B) e^{(t+h-\tau) B} \operatorname{Pf}(\tau)\right. \\
& \left.-(1-B) e^{(t-\tau) B} \operatorname{Pf}(\tau)\right] \mathrm{d} \tau \\
& +\int_{t}^{t+h}(1-B) e^{(t+h-\tau) B} \operatorname{Pf}(\tau) \mathrm{d} \tau \|_{\eta, p} \leq M h^{\theta-\eta-1 / r} .
\end{aligned}
$$

\section{Existence and uniqueness for the stochastic Volterra equation}

\subsection{Preliminaries on stochastic integration theory in Banach spaces}

Since the theory of stochastic integration in Banach spaces is relatively recent, we present here a short introduction to its main steps.

Fixed a Banach space $E$, we are interested in the construction of the stochastic integral $\int \Phi(s) \mathrm{d} W(s)$, where $\Phi$ is an adapted process with values in a space of linear operators from $U$ into $E$ and $W$ is a $U$-valued cylindrical Wiener process. In this section we give a concise introduction to the relevant construction.

The starting point is the choice of the space $E$. One of the reasons for the recent interest in UMD spaces is that they are those Banach spaces where the Wiener integral of $\mathcal{L}(U, E)$-valued functions can be extended to Itô integral of $\mathcal{L}(U, E)$-valued stochastic processes, see the seminal papers by van Neerven et al. [28].

As an example, the class of UMD spaces contains the $L^{p}$-spaces for $1<$ $p<\infty$. However, we shall restrict ourselves to the case of spaces of type 2 , see the characterization in [17], which contains all Hilbert spaces and the $L^{p_{-}}$ spaces for $2 \leq p<\infty$. The following well known properties imply that the state space $X$ defined in Sect. 3 inherits these properties from $E$.

Remark 5.1. Let $E$ be a Banach space, $-A$ a sectorial operator on $E$. Let $(S, \Sigma, \mu)$ be a $\sigma$-finite measure space.

(1) If $E$ is a UMD space and $p \in(1, \infty)$, then $\mathbf{L}^{p}(S ; E)$ and $(E, D(A))_{\theta, p}$ are also UMD spaces.

(2) If $E$ is of type 2 and $2 \leq p<\infty$, then also $\mathbf{L}^{p}(S ; E)$ and $(E, D(A))_{\theta, p}$ are of type 2 .

Proof. It is well known and easily seen that $\mathbf{L}^{p}(S ; E)$ inherits the UMD property and the type from $E$. Since $x \mapsto t^{\theta}(1-A) R(\cdot, A) x$ is an isometric isomorphism from $E_{\theta, p}$ into a closed subspace of $\mathbf{L}^{p}\left((1, \infty) ; t^{-1} \mathrm{~d} t ; E\right)$, the interpolation space inherits type and UMD property as well. (See also [23, Proposition 1]).

We need to recall the notion of $\gamma$-radonifying operators. As it happens for UMD spaces with respect to Hilbert spaces, also this class of operators 
can be introduced as the natural extension in the Banach setting of the Hilbert-Schmidt operators. By definition, an operator $R: H \rightarrow E$ ( $H$ separable Hilbert space, $E$ Banach space) is $\gamma$-radonifying if for some (and hence every) orthonormal basis $\left\{e_{k}\right\}$ in $H$ the Gaussian sum $\sum_{n=1}^{\infty} \gamma_{n} R e_{n}$ converges in $L^{2}(\Omega ; E)$. Here, $\gamma=\left\{\gamma_{n}\right\}$ is a sequence of independent real standard Gaussian random variables. The space $\gamma(H, E)$ of $\gamma$-radonifying operators from $H$ to $E$ is a Banach space with respect to the norm

$$
\|R\|_{\gamma(H, E)}^{2}=\mathbb{E}\left\|\sum_{n=1}^{\infty} \gamma_{n} R e_{n}\right\|^{2}
$$

The norm is independent of the basis $\left\{e_{n}\right\}$; further, given linear bounded operators $S: H^{\prime} \rightarrow H$ and $T: E \rightarrow E^{\prime}$ and $R \in \gamma(H, E)$, then $T R S \in \gamma\left(H^{\prime}, E^{\prime}\right)$ and it holds

$$
\|T R S\|_{\gamma\left(H^{\prime}, E^{\prime}\right)} \leq\|T\|\|R\|_{\gamma(H, E)}\|S\| .
$$

The stochastic integral in a UMD space $E$ with respect to a $U$-valued cylindrical Wiener process was constructed in the paper [25] by van Neerven et al.; here we recall the basics of the construction.

Let $\mathcal{E}$ be the set of $\mathcal{F}_{t}$-adapted finite rank processes in $\gamma(U, E)$, i.e., $g \in \mathcal{E}$ means that

$$
G(t, \omega)=\sum_{i=1}^{n} 1_{\left(0, t_{i}\right] \times F_{i}}(t, \omega)\left(h_{i} \otimes x_{i}\right),
$$

with $t_{i} \in \mathbb{R}_{+}, F_{i} \in \mathcal{F}_{t_{i}}, x_{i} \in E, h_{i} \in U$. The stochastic integral of a process $G \in \mathcal{E}$ is defined by

$$
\int G(s) \mathrm{d} W(s)=\sum_{i=1}^{n} 1_{F_{i}}(\omega)\left[W\left(t_{i}\right) h_{i}\right] \otimes x_{i} .
$$

Note. In the sequel, we denote $A \asymp B$ if there exists constants such that $c_{1} A \leq B \leq c_{2} A$.

Proposition 5.2. Let $E$ be a UMD space and fix $G \in \mathcal{E}$. Then for every $p \in$ $(1, \infty)$ it holds

$$
\mathbb{E} \sup _{t \in[0, T]}\left\|\int_{\mathbb{R}_{+}} G(s) \mathrm{d} W(s)\right\|^{p} \asymp \mathbb{E}\|G\|_{\gamma\left(L^{2}\left(\mathbb{R}_{+} ; U\right), E\right)}^{p}
$$

with constants depending only on $p$ and $E$.

Using (5.1), the stochastic integral can be then extended to the closure $L_{\mathcal{F}}^{p}\left(\Omega ; \gamma\left(L^{2}\left(\mathbb{R}_{+} ; U\right), E\right)\right)$ of $\mathcal{E}$ in $L^{p}\left(\Omega ; \gamma\left(L^{2}\left(\mathbb{R}_{+} ; U\right), E\right)\right)$. For Banach type 2 spaces, we have the continuous embedding $L^{2}\left(\mathbb{R}_{+} ; \gamma(U, E)\right) \hookrightarrow$ $\gamma\left(L^{2}\left(\mathbb{R}_{+} ; H\right), E\right)$ which implies, for $\Phi: \mathbb{R}_{+} \rightarrow \gamma(U, E)$ an adapted and strongly measurable process,

$$
\mathbb{E}\left\|\int_{\mathbb{R}_{+}} \Phi(s) \mathrm{d} W(s)\right\|^{p} \leq C \mathbb{E}\left|\int_{\mathbb{R}_{+}}\|\Phi(t)\|_{\gamma(U, E)}^{2} \mathrm{~d} t\right|^{p / 2}<\infty .
$$


Proposition 5.3. By a density argument and using the above isometry formulas we obtain that the stochastic integral uniquely extends to $L_{\mathcal{F}}^{p}\left(\Omega ; L^{2}\left(\mathbb{R}_{+} ; \gamma(U\right.\right.$, $E))$ ) the closed linear span in $L^{p}\left(\Omega ; L^{2}\left(\mathbb{R}_{+} ; \gamma(U, E)\right)\right)$ of all processes in $\mathcal{E}$.

\subsection{Stochastic convolution and the mild solution in state space}

Let us recall the state space setting from previous sections. Since $E$ is a UMD space of type 2 , then $X$ is a UMD space of type 2 by Remark 5.1 ; we are given on $X$ a linear unbounded operator $B: D(B) \subset X \rightarrow X$ that is the generator of an analytic semigroup, with its real interpolation spaces $X_{\theta, p}$. Let $0<\eta<\theta<1$ with $\theta-\eta>\frac{1}{2}$. We let $P: H \rightarrow X_{\theta, p}$ (with $\theta>\frac{1}{2}$ ) be a bounded linear operator and $v_{0}: \Omega \rightarrow X$ be a random variable. Then we consider the problem

$$
\begin{aligned}
\mathrm{d} v(t) & =B(v(t) \mathrm{d} t-P \Phi(t) \mathrm{d} W(t))+P \Phi(t) \mathrm{d} W(t), \\
v(0) & =v_{0} .
\end{aligned}
$$

For shorthand we set

$$
\Psi(t):=(1-B) P \Phi(t) \in \mathcal{L}\left(U, X_{\theta-1, p}\right),
$$

and define the stochastic convolution process

$$
W_{S}(t)=e^{t B} v_{0}+\int_{0}^{t} e^{(t-r) B} \Psi(r) \mathrm{d} W(r) .
$$

We shall write the solution of Problem (5.3) in the mild form

$$
v(t)=e^{t B} v_{0}+W_{S}(t) .
$$

Proposition 5.4. Let $T>0$ be given. Then the stochastic convolution process $\left\{W_{S}(t), t \in[0, T]\right\}$ has, almost surely, continuous trajectories with values in the interpolation space $X_{\eta, p}$.

Proof. We first notice that the isometry formula (5.2), which holds for $p \geq 2$, allows to use Kolmogorov's test in order to prove the desired regularity result for the stochastic convolution process.

Let $\delta$ be given in assumption (2.9) and let $\beta=\delta-1+(\theta-\eta)$. We write, for a constant $C$ which may depend on $p$ and may vary from line to line,

$$
\begin{aligned}
\mathbb{E}\left\|W_{S}(t)-W_{S}(\tau)\right\|_{\eta, p}^{p} \leq & \left.C \mathbb{E} \| \int_{0}^{\tau}\left[e^{(t-r) B}-e^{(\tau-r) B}\right)\right] \Psi(r) \mathrm{d} W(r) \|_{\eta, p}^{p} \\
& +C \mathbb{E}\left\|\int_{\tau}^{t} e^{(t-r) B} \Psi(r) \mathrm{d} W(r)\right\|_{\eta, p}^{p}=I_{1}+I_{2}
\end{aligned}
$$

and we estimate

$$
\begin{aligned}
I_{1} \leq & C \mathbb{E}\left\|\left(e^{(t-\tau) B}-I\right) e^{(\tau-\cdot) B} \Psi(\cdot)\right\|_{L^{2}\left(0, \tau ; \gamma\left(U, X_{\eta, p}\right)\right)}^{p} \\
\leq & C\left\|\left(e^{(t-\tau) B}-I\right)(I-B)^{-\beta}\right\|_{\mathcal{L}\left(X_{\eta, p}, X_{\eta, p}\right)}^{p} \\
& \times \mathbb{E}\left|\int_{0}^{\tau}\left\|(I-B)^{\beta} e^{(\tau-r) B} \Psi(r)\right\|_{\gamma\left(U, X_{\eta, p}\right)}^{2} \mathrm{~d} r\right|^{p / 2} .
\end{aligned}
$$


Notice that $B$ generates an analytic semigroup hence the following estimate holds:

$$
\sup _{t>0} t^{-\beta}\left\|(I-B)^{-\beta}\left(e^{t B}-I\right)\right\|<\infty .
$$

We concentrate on the last term of the estimate for $I_{1}$ :

$$
\begin{aligned}
& \mathbb{E}\left|\int_{0}^{\tau}\left\|(I-B)^{\beta} e^{(\tau-r) B} \Psi(r)\right\|_{\gamma\left(U, X_{\eta, p}\right)}^{2} \mathrm{~d} r\right|^{p / 2} \\
& \quad \leq \mathbb{E}\left|\int_{0}^{\tau}\left\|e^{(\tau-r) B}(I-B)^{1+\beta}\right\|_{\mathcal{L}\left(X_{\theta, p}, X_{\eta, p}\right)}^{2}\|P\|_{\mathcal{L}\left(E, X_{\theta, p}\right)}^{2}\|\Phi(r)\|_{\gamma(U, E)}^{2} \mathrm{~d} r\right|^{p / 2}
\end{aligned}
$$

by [21, Prop. 2.2.7 and Prop. 2.3.1] we get the bound $\| r^{1+\beta+\eta-\theta} e^{r B}(I-$ $B)^{1+\beta} \|_{\mathcal{L}\left(X_{\theta, p}, X_{\eta, p}\right)}^{2} \leq C$ and we obtain

$$
\begin{aligned}
& \mathbb{E}\left|\int_{0}^{\tau}\left\|(I-B)^{\beta} e^{(\tau-r) B} \Psi(r)\right\|_{\gamma\left(U, X_{\eta, p}\right)}^{2} \mathrm{~d} r\right|^{p / 2} \\
& \quad \leq C \mathbb{E}\left|\int_{0}^{\tau}\left\|(\tau-r)^{\theta-\eta-1-\beta} \Phi(r)\right\|_{\gamma(U, E)}^{2} \mathrm{~d} r\right|^{p / 2}
\end{aligned}
$$

and we see that this quantity is finite by using assumption (2.9) on $\Phi$. By recollecting the above estimates we get therefore

$$
I_{1} \leq C(t-\tau)^{p \beta}
$$

We now turn to $I_{2}$. We have

$$
\begin{aligned}
& \mathbb{E}\left\|\int_{\tau}^{t} e^{(t-r) B} \Psi(r) \mathrm{d} W(r)\right\|_{\eta, p}^{p} \leq C \mathbb{E}\left\|e^{(t-\cdot) B} \Psi(\cdot)\right\|_{L^{2}\left(\tau, t ; \gamma\left(U, X_{\eta, p}\right)\right)}^{p} \\
& =\mathbb{E}\left|\int_{\tau}^{t}(\tau-r)^{2 \beta}\left\|(\tau-r)^{1+\eta-\theta} e^{(\tau-r) B}(I-B) P\right\|_{\mathcal{L}\left(E, X_{\eta, p}\right)}^{2}\left\|(\tau-r)^{\theta-\eta-1-\beta} \Phi(r)\right\|_{\gamma(U, E)}^{2} \mathrm{~d} r\right|^{p / 2}
\end{aligned}
$$

and, similarly to the first term, we get

$$
I_{2} \leq C(t-\tau)^{p \beta}
$$

We have proved that

$$
\mathbb{E}\left\|W_{S}(t)-W_{S}(\tau)\right\|_{\eta, p}^{p} \leq C(t-\tau)^{p \beta} ;
$$

we recall that the assumption $\delta>\frac{1}{p}+1-(\theta-\eta)$ and the setting $\beta=\delta-1+(\theta-\eta)$ implies $\beta p>1$. Therefore estimate (5.4) implies, by Kolmogorov's continuity theorem, that there exists a version of the stochastic convolution process with almost surely continuous trajectories, which are even locally Hölder continuous for arbitrary exponent less than $\frac{\beta p-1}{p}$.

It is known from Proposition 3.8 that $B$ is the generator of a holomorphic semigroup $e^{t B}$ such that, for all $\epsilon>0$, we have that $\lim _{t \rightarrow \infty} e^{-\epsilon t}\left\|e^{t B}\right\|=0$. The following proposition shows that the stochastic convolution process takes values, in $p$-mean, in an interpolation space of higher regularity than just $X_{\eta, p}$. 
In order to handle the behaviour at infinity we shall introduce the weighted space $L^{p}\left(\mathbb{R}_{+}, e^{-\epsilon t} \mathrm{~d} t ; X\right)$ of $X$-valued functions such that

$$
\int_{\mathbb{R}_{+}}\|f(t)\|^{p} e^{-\epsilon t} \mathrm{~d} t<+\infty .
$$

Moreover, $L_{\mathcal{F}}^{p}\left(\mathbb{R}_{+}, e^{-\epsilon t} \mathrm{~d} t ; X\right)$ is the space of $X$-valued processes, adapted to the filtration $\left\{\mathcal{F}_{t}\right\}$, endowed with the norm

$$
\|\Phi\|_{L_{\mathcal{F}}^{p}\left(\mathbb{R}_{+}, e^{-\epsilon t} \mathrm{~d} t ; X\right)}^{p}=\mathbb{E} \int_{\mathbb{R}_{+}}\|\Phi(t)\|^{p} e^{-\epsilon t} \mathrm{~d} t .
$$

Proposition 5.5. Assume that for some $\epsilon>0$ it holds

$$
\mathbb{E} \int_{0}^{\infty}\|\Phi(t)\|_{\gamma(U, E)}^{p} e^{-\epsilon t} \mathrm{~d} t<+\infty
$$

Then, the process $t \mapsto e^{-\epsilon t} W_{S}(t)$ belongs to $L_{\mathcal{F}}^{p}\left(\mathbb{R}_{+}, e^{-\epsilon t} \mathrm{~d} t ; X_{\eta, p}\right)$ for any $\eta<\theta-\frac{1}{2}$ and we estimate

$$
\mathbb{E} \int_{0}^{\infty}\left\|W_{S}(t)\right\|_{\eta, p}^{p} e^{-\epsilon t} \mathrm{~d} t \leq C \mathbb{E} \int_{0}^{\infty}\|\Phi(s)\|_{\gamma(U, E)}^{p} e^{-\epsilon t} \mathrm{~d} t .
$$

Proof. We put $\delta=\epsilon / p$ and estimate

$$
\begin{aligned}
\mathbb{E} & \left\|e^{-\epsilon t} W_{S}(t)\right\|_{\eta, p}^{p}=\mathbb{E}\left\|\int_{0}^{t} e^{-\delta t}(1-B) e^{(t-s) B} P \Phi(s) \mathrm{d} W(s)\right\|_{\eta, p}^{p} \\
& \leq \mathbb{E}\left[\int_{0}^{t}\left(e^{-\delta(t-s)}\left\|(1-B) e^{(t-s) B}\right\|_{\mathcal{L}\left(X_{\theta, p}, X_{\eta, p}\right)}\|P\|_{\mathcal{L}\left(E, X_{\theta, p}\right)} e^{-\delta s}\|\Phi(s)\|_{\gamma(U, E)}\right)^{2} \mathrm{~d} s\right]^{\frac{p}{2}} \\
& \leq C \mathbb{E}\left[\int_{0}^{t} e^{-2 \delta(t-s)}(t-s)^{2(\theta-\eta-1)} e^{-2 \delta s}\|\Phi(s)\|_{\gamma(U, E)}^{2} \mathrm{~d} s\right]^{p / 2} \\
& =C \mathbb{E}\left[g * \left(e^{\left.\left.-2 \delta \cdot\|\Phi(\cdot)\|_{\gamma(U, E)}\right)(t)\right]^{\frac{p}{2}}}\right.\right.
\end{aligned}
$$

with $g(t):=e^{-2 \delta t} t^{2(\theta-\eta-1)}$. Since $\theta-\eta-1>-\frac{1}{2}$, we infer that $g \in$ $\mathbf{L}^{1}([0, \infty), \mathbb{R})$. Therefore convolution by $g$ maps $\mathbf{L}^{p / 2}([0, \infty) ; \mathbb{R})$ continuously into $\mathbf{L}^{p / 2}([0, \infty) ; \mathbb{R})$. Hence

$$
\begin{aligned}
& \mathbb{E} \int_{0}^{\infty} e^{-\epsilon t}\left\|W_{S}(t)\right\|_{\eta, p}^{p} \mathrm{~d} t \leq C \mathbb{E} \int_{0}^{\infty}\left[g *\left(e^{-2 \delta} \cdot\|\Phi(\cdot)\|_{\gamma(U, E)}\right)\right]^{\frac{p}{2}}(t) \mathrm{d} t
\end{aligned}
$$

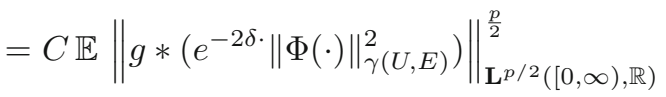

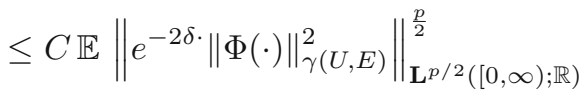

$$
\begin{aligned}
& =C \mathbb{E} \int_{0}^{\infty} e^{-\epsilon t}\|\Phi(t)\|_{\gamma(U, E)}^{p} \mathrm{~d} t
\end{aligned}
$$

Remark 5.6. We notice that the assumption in Proposition 5.5 holds whenever $\Phi$ satisfies Hypothesis 2.10. 


\subsection{Laplace transform for stochastic processes}

In this section we examine the Laplace transform of the stochastic process $v(t)$ that is the mild solution of problem (5.3). First we introduce the Laplace transform of a stochastic integral.

Definition 5.7. We are given the cylindrical Wiener process $\{W(t)\}_{t \geq 0}$ on the Hilbert space $U$; we let $\Psi_{1}, \Psi_{2}$ be adapted stochastic processes, with values in $\mathcal{L}(U, K)$ and $\gamma(U, K)$ respectively, such that

$$
\begin{gathered}
\mathbb{E} \int_{0}^{\infty}\left\|e^{-\omega t} \Psi_{1}(t)\right\|_{\mathcal{L}(U, K)}^{2} \mathrm{~d} t<+\infty \\
\mathbb{E} \int_{0}^{\infty}\left\|e^{-\omega t} \Psi_{2}(t)\right\|_{\gamma(U, K)}^{2} \mathrm{~d} t<+\infty
\end{gathered}
$$

for some $\omega \in \mathbb{R}$. Then we define the processes

$$
\begin{aligned}
\mathcal{L}\left[\Psi_{1}\right](s) & =\int_{0}^{\infty} e^{-s t} \Psi_{1}(t) \mathrm{d} t \\
\mathcal{L}\left[\Psi_{2} \mathrm{~d} W\right](s) & =\int_{0}^{\infty} e^{-s t} \Psi_{2}(t) \mathrm{d} W(t), \quad s \geq \omega .
\end{aligned}
$$

Remark 5.8. Let $z(t)=\mathcal{L}[\Psi \mathrm{d} W](t)$ be defined as above.

(1) The process $z(t)$ is not adapted to $\left\{\mathcal{F}_{t}\right\}$.

(2) $z(t) \in L^{2}(\Omega, \mathcal{F} ; K)$ for every $t \geq \omega$.

(3) $z$ admits a continuous modification.

Proof. (1) is obvious and (2) is an easy application of Ito's isometry. To prove (3) notice that for $\omega \leq s<t$

$$
z(t)-z(s)=\int_{0}^{\infty}\left[e^{-t x}-e^{-s x}\right] \Psi(x) \mathrm{d} W(x) .
$$

Therefore

$$
\begin{aligned}
\mathbb{E}\|z(t)-z(s)\|_{K}^{2} & =\mathbb{E}\left\|\int_{0}^{\infty}\left[e^{-t x}-e^{-s x}\right] \Psi(x) \mathrm{d} W(x)\right\|_{K}^{2} \\
& =\mathbb{E} \int_{0}^{\infty}\left\|\int_{s}^{t} x e^{-\tau x} \mathrm{~d} \tau \Psi(x)\right\|_{\gamma(U, K)}^{2} \mathrm{~d} x \\
& \leq \mathbb{E} \int_{0}^{\infty} x^{2} e^{-2 s x}(t-s)^{2}\|\Psi(x)\|_{\gamma(U, K)}^{2} \mathrm{~d} x \leq C(t-s)^{2},
\end{aligned}
$$

where $C=\sup _{x \in \mathbb{R}_{+}} x^{2} e^{-2(s-\omega) x}<+\infty$, which implies, by Kolmogorov's continuity criterium, the existence of a continuous modification of $z(t)$.

The following remark is a straightforward consequence of the stochastic Fubini Theorem, see for instance [12] for the Hilbert case and [24] for the Banach case. We consider stochastic processes $\Psi_{1}$ and $\Psi_{2}$ as in Definition 5.7. Then the convolution formula for Laplace transforms holds:

$$
\mathcal{L}\left[\int_{0}^{t} \Psi_{1}(t-\tau) \Psi_{2}(\tau) \mathrm{d} W(\tau)\right](s)=\mathcal{L}\left[\Psi_{1}\right](s) \mathcal{L}\left[\Psi_{2} \mathrm{~d} W\right](s), \quad s>2 \omega .
$$




\subsection{The solution to the stochastic integral equation}

Let us first justify that initial functions in $\tilde{X}_{\mu, \delta}$ lead to initial states $v_{0} \in X_{\eta, p}$ for suitable $\eta$.

Lemma 5.9. Let $A$ and a satisfy Hypotheses 2.1 and 2.4 with $\alpha(a)>\frac{1}{2}$. Let $\mu>\frac{1}{p}$ and $\delta>2-\alpha(a)$. Let $P, Q, J$ be defined as in Sect. 3. Then there exist $0<\eta<\theta<1$ such that $P: E \rightarrow X_{\theta, p}, J: X_{\eta, p} \rightarrow E$, and $Q: \tilde{X}_{\mu, \delta} \rightarrow X_{\eta, p}$ are continuous, and $\theta-\eta>\frac{1}{2}$.

Proof. Lemmas 3.12 and 3.14 guarantee continuity of $P: E \rightarrow X_{\theta, p}$ and $J: X_{\eta, p} \rightarrow E$ if

$$
\theta<\theta_{0}:=\frac{1}{p}+\frac{\alpha(a)}{q}, \quad \eta>\eta_{0}:=\frac{1}{p}-\frac{\alpha(a)}{p} .
$$

To conclude continuity of $Q$ we check the assumptions of Lemma 3.16(3). So we have to find $\eta>\eta_{0}$ such that the following conditions hold:

$$
\begin{aligned}
\eta-\eta_{0} & <\alpha(a) \mu+\frac{1-\alpha(a)}{p}-\eta_{0}=\alpha(a) \mu . \\
\eta-\eta_{0} & <\frac{\delta-1}{q}-\eta_{0}=\frac{\delta}{q}-\frac{1}{q}-\frac{1}{p}+\frac{\alpha(a)}{p} \\
& =\frac{1}{q}\left(\delta-1-\frac{q}{p}(1-\alpha(a))\right)=\frac{1}{q}(\delta-1-(q-1)(1-\alpha(a))) . \\
\eta-\eta_{0} & <\delta-1-\frac{1-\alpha(a)}{q}-\eta_{0} \\
& =\delta-1-\frac{1}{q}(1-\alpha(a))-\frac{1}{p}(1-\alpha(a))=\delta-2+\alpha(a) .
\end{aligned}
$$

Evidently, the right side of (5.8) is strictly positive. Since $\delta>2-\alpha(a)$, the right hand side of (5.10) is strictly positive. Finally, since $p \geq 2$ we have $q \leq 2$, and

$$
\delta-1-(q-1)(1-\alpha(a)) \geq \delta-1-(1-\alpha(a))=\delta-2+\alpha(a)>0 .
$$

Therefore, one needs only to choose $\eta$ sufficiently close to $\eta_{0}$ to ensure continuity of $Q$. Finally, since $\theta_{0}-\eta_{0}=\alpha(a)>\frac{1}{2}$, we can obtain $\theta-\eta>\frac{1}{2}$ by chosing $\eta$ close to $\eta_{0}$ and $\theta$ close to $\theta_{0}$.

Uniqueness of the weak solution can be inferred from the deterministic theory, compare for instance [20]:

Lemma 5.10. In our setting, let $u_{0} \in L^{2}\left(\Omega, \mathcal{F}_{0} ; \tilde{X}_{\mu, \delta}\right)$. Then there exists at most one weak solution to equation (1.2).

Proof. In fact, if $u_{1}, u_{2}$ are two weak solutions of problem (1.2), then $u=$ $u_{2}-u_{1}$ is a weak solution to the deterministic problem

$$
\begin{aligned}
& \frac{d}{d t} \int_{-\infty}^{t} a(t-s) u(s) d s=A u(t), \\
& u(s)=0 \quad \text { for } s \leq 0 .
\end{aligned}
$$


Then we can resort on the uniqueness of the solution proved in Section 4 which implies that $u=0$.

Now we can prove the existence of solution for the the stochastic integral equation (1.2).

Proof of Theorem 2.14. Remember that by assumption $\eta<\theta-\frac{1}{2}$. By Lemma 3.13 the operator $J_{0}$ can be extended to a bounded operator $J$ : $X_{\eta, p} \rightarrow E$. By Proposition 5.5 we know that estimate (2.10) holds for some $\epsilon>0$. We use a a Laplace transform argument. First we compute

$$
\begin{aligned}
\mathcal{L}\left[\int_{-\infty}^{0} a(t-\tau) u_{0}(\tau) \mathrm{d} \tau\right](s) & =\int_{0}^{\infty} \int_{-\infty}^{0} \int_{[0, \infty)} e^{-s t} e^{-\kappa(t-\tau)} u_{0}(\tau) \nu(\mathrm{d} \kappa) \mathrm{d} \tau \mathrm{d} t \\
& =\int_{[0, \infty)}^{0} \int_{-\infty}^{0} \frac{1}{s+\kappa} e^{\kappa \tau} u_{0}(\tau) \mathrm{d} \tau \nu(\mathrm{d} \kappa) \\
& =\int_{[0, \infty)} \frac{1}{s+\kappa} v_{0}(\kappa) \nu(\mathrm{d} \kappa)
\end{aligned}
$$

and

$\bar{u}=\int_{-\infty}^{0} a(-s) u_{0}(s) \mathrm{d} s=\int_{-\infty}^{0} \int_{[0, \infty)} e^{\kappa s} u_{0}(s) \nu(\mathrm{d} \kappa) \mathrm{d} s=\int_{[0, \infty)} v_{0}(\kappa) \nu(\mathrm{d} \kappa)$.

Taking Laplace transforms in (2.12), we have

$$
\mathcal{L} v(s)=R(s, B) v_{0}+(1-B) R(s, B) P \mathcal{L}[\Phi \mathrm{d} W](s) .
$$

Using Lemmas 3.5 and 3.11 we obtain

$$
\begin{aligned}
\mathcal{L}[J v](s) & =J R(s, B) v_{0}+J(1-B) R(s, B) P \mathcal{L}[\Phi \mathrm{d} W](s) \\
& =R(s \hat{a}(s), A) \int_{[0, \infty)} \frac{\kappa}{s+\kappa} v_{0}(\kappa) \nu(\mathrm{d} \kappa)+R(s \hat{a}(s), A) \mathcal{L}[\Phi \mathrm{d} W](s) .
\end{aligned}
$$

Now pick any $\zeta \in D\left(A^{*}\right)$. Then we have (in the following computation, we denote $\langle\xi, x\rangle$ the action of $\xi \in E^{*}$ on $\left.x \in E\right)$ that

$$
\begin{aligned}
\mathcal{L}\left[\int_{-\infty}^{t}\langle\zeta, a(t-\tau) u(\tau)\rangle \mathrm{d} \tau-\langle\zeta, \bar{u}\rangle-\int_{0}^{t}\left\langle A^{*} \zeta, u(\tau)\right\rangle \mathrm{d} \tau-\int_{0}^{t}\langle\zeta, \Phi(\tau) \mathrm{d} W(\tau)\rangle\right](s) \\
=\mathcal{L}\left[\left\langle\int_{-\infty}^{0} \zeta, a(t-\tau) u_{0}(\tau) \mathrm{d} \tau\right\rangle-\langle\zeta, \bar{u}\rangle\right](s) \\
+\mathcal{L}\left[\left\langle\zeta, \int_{0}^{t} a(t-\tau) J v(\tau) \mathrm{d} \tau\right\rangle-\left\langle A^{*} \zeta, \int_{0}^{t} J v(\tau)\right\rangle \mathrm{d} \tau\right](s) \\
\quad-\mathcal{L}\left[\int_{0}^{t}\langle\zeta, \Phi(\tau) \mathrm{d} W(\tau)\rangle\right](s) \\
=\left\langle\zeta, \int_{[0, \infty)} \frac{1}{s+\kappa} v_{0}(\kappa) \nu(\mathrm{d} \kappa)\right\rangle-\left\langle\zeta, \frac{1}{s} \int_{[0, \infty)} v_{0}(\kappa) \nu(\mathrm{d} \kappa)\right\rangle \\
+\left\langle\left(\hat{a}(s)-\frac{1}{s} A^{*}(s)\right) \zeta, R(s \hat{a}(s), A) \int_{[0, \infty)} \frac{\kappa}{s+\kappa} v_{0}(\kappa) \nu(\mathrm{d} \kappa)\right.
\end{aligned}
$$




$$
\begin{aligned}
& +R(s \hat{a}(s), A) \mathcal{L}[\Phi \mathrm{d} W](s)\rangle \\
& -\left\langle\zeta, \frac{1}{s} \mathcal{L}[\Phi \mathrm{d} W](s)\right\rangle \\
= & \left\langle\zeta, \int_{[0, \infty)}-\frac{\kappa}{s(s+\kappa)} v_{0}(\kappa) \nu(\mathrm{d} \kappa)\right\rangle \\
& +\left\langle\zeta, \frac{1}{s} \int_{[0, \infty)} \frac{\kappa}{s+\kappa} v_{0}(\kappa) \nu(\mathrm{d} \kappa)+\frac{1}{s} \mathcal{L}[\Phi \mathrm{d} W](s)\right\rangle \\
& -\left\langle\zeta, \frac{1}{s} \mathcal{L}[\Phi \mathrm{d} W](s)\right\rangle \\
= & 0 .
\end{aligned}
$$

Thus $u$ satisfies the identity (2.11) and it is a weak solution of problem (1.2) as required.

\section{Long time behavior}

In this section we concentrate on the case of a constant covariance operator $\Phi$, i.e., we require that Hypothesis 2.10(2) holds. In this setting, we study the long time behaviour of the Markov process $v(t)$ that is the solution of problem (5.3).

Let us recall that $v(t)=e^{t B} v_{0}+W_{S}(t)$ inherits its regularity from the initial condition $v_{0}$ and the stochastic convolution process $W_{S}(t)$. The latter is, according to Proposition 5.4, a stochastic process with continuous paths in the interpolation space $X_{\eta, p}$.

Further, we shall impose that the semigroup $e^{t B}$ is of negative type. Proposition 3.9 gives conditions on the original problem (1.2) to guarantee this.

According to Proposition 5.4, we introduce the transition semigroup $\mathcal{P}_{t}$ on the space $C_{b}\left(X_{\eta, p}\right)\left(\eta<\theta-\frac{1}{2}\right)$ of real valued bounded continuous functions on $X_{\eta, p}$, defined by

$$
\mathcal{P}_{t} f(x)=\mathbb{E}\left[f\left(e^{t B} x+W_{S}(t)\right)\right], \quad t \geq 0, \quad x \in X_{\eta}, \quad f \in C_{b}\left(X_{\eta, p}\right) .
$$

A Radon measure $\mu$ on $X_{\eta, p}$ is said to be an invariant measure for problem (5.3) (or, equivalently, for the transition semigroup $\mathcal{P}_{t}$ ), if

$$
\int_{X_{\eta, p}} \mathcal{P}_{t} f(x) \mathrm{d} \mu=\int_{X_{\eta, p}} f(x) \mathrm{d} \mu \quad \forall t>0, \quad f \in C_{b}\left(X_{\eta, p}\right) .
$$

Existence and uniqueness of invariant measure for a stochastic evolution equation in Hilbert spaces is a classical problem and we can refer to the monograph by Da Prato and Zabczyk [12] for a complete discussion of the matter. Recently it was realized that several parts of the theory can be transferred to the Banach space setting with minor changes, see, e.g., the papers [16,27].

We first remark that, whenever the covariance operator $\Phi$ is given in the space $\gamma(U, E)$, then the stochastic convolution process is a Gaussian process in $L_{\mathcal{F}}^{2}\left((0, T) \times \Omega ; X_{\eta, p}\right)$ with zero mean and covariance operator $Q_{t}$ defined by 


$$
\left\langle Q_{t} x^{*}, y^{*}\right\rangle=\int_{0}^{t}\left\langle e^{r B} \Psi \Psi^{*} e^{r B^{*}} x^{*}, y^{*}\right\rangle \mathrm{d} r, \quad x^{*}, y^{*} \in X_{\eta, p}^{*}, \quad t>0 .
$$

(In the above formula we use the shorthand notation $\Psi=(I-B) P \Phi \in$ $\mathcal{L}\left(E, X_{\theta-1, p}\right)$.)

Then the main result of this section is a consequence of the characterization theorem [27, Proposition 4.4] that extends, to the Banach space setting, the corresponding results of [12, Theorem 11.7]. For the sake of clarity, we recall it here.

Proposition 6.1. ([27], Proposition 4.4, Corollary 4.3)

(1) Assume that the function $t \mapsto e^{t B} \Psi(t)$ belongs to $\gamma\left(\mathbf{L}^{2}\left(\mathbb{R}_{+} ; U\right) ; X_{\eta, p}\right)$. Then there exists an invariant measure $\mu$ for the transition semigroup $\mathcal{P}_{t}$.

(2) Assume that $e^{t B^{*}} x^{*} \rightarrow 0$ in the weak $k^{*}$-topology for $x^{*}$ in a weak ${ }^{*}$-sequentially dense subspace of $X_{\eta}^{*}$. Then there exists at most one invariant measure $\mu$ for the transition semigroup $\mathcal{P}_{t}$, and it is the centered Gaussian measure with the covariance operator

$$
Q_{\infty}=\lim _{t \rightarrow \infty} Q_{t} \in \mathcal{L}\left(X_{\eta, p}^{*}, X_{\eta, p}\right) .
$$

Theorem 6.2. Suppose that Hypotheses 2.1, 2.4, 2.10(2), and 2.9 hold, let $X, B, P, Q, J$ be defined as in Sect. 3 , and suppose that $e^{\omega t}\left\|e^{t B}\right\| \rightarrow 0$ for some $\omega>0$.

Then there exists a unique invariant measure for the transition semigroup $\mathcal{P}_{t}$ related to Problem (5.3).

Proof. We show existence of the invariant measure using Proposition 6.1(1). Since $X_{\eta, p}$ is of type 2 , we need only to show that $e^{t B} \Psi \in \mathbf{L}^{2}\left([0, \infty), \gamma\left(U ; X_{\eta, p}\right)\right)$. Take some $\epsilon<\omega$. Then

$$
\left\|e^{t B}\right\|_{\mathcal{L}\left(X_{\theta-1, p} ; X_{\eta, p}\right)} \leq C t^{\theta-1-\eta} e^{-\epsilon t} .
$$

Thus (using the ideal property of $\gamma(U ; E)$ )

$$
\begin{aligned}
& \left\|e^{t B} P(1-B) \Phi\right\|_{\gamma\left(U ; X_{\eta, p}\right)} \\
& \quad \leq\left\|e^{t B}\right\|_{\mathcal{L}\left(X_{\theta-1, p}, X_{\eta, p}\right)}\|(1-B)\|_{\mathcal{L}\left(X_{\theta} ; X_{\theta-1, p}\right)}\|P\|_{\mathcal{L}\left(E ; X_{\theta, p}\right)}\|\Phi\|_{\gamma(U ; E)} \\
& \quad \leq C t^{\theta-\eta-1} e^{-\epsilon t} .
\end{aligned}
$$

Since $\theta-\eta-1>-\frac{1}{2}$, we have $t^{\theta-\eta-1} e^{-\epsilon t} \in \mathbf{L}^{2}([0, \infty) ; \mathbb{R})$.

Uniqueness of the invariant measure follows from Proposition 6.1(2) and the fact that $\left\|e^{t B^{*}}\right\| \rightarrow 0$.

Existence and uniqueness of the invariant measure $\mu$ for the transition semigroup $\mathcal{P}_{t}$ related to Problem (5.3) implies, in particular, that the measure is ergodic and strongly mixing: for every $\phi \in C_{b}\left(X_{\eta, p}\right)$ it holds

$$
\lim _{t \rightarrow+\infty} \mathbb{E}\left[\phi\left(v\left(t ; v_{0}\right)\right)\right]=\int_{X_{\eta, p}} \phi(\xi) \mu(\mathrm{d} \xi) .
$$


Corollary 6.3. Under the assumptions of Theorem 6.2, for every $\psi \in C_{b}(H)$ we have

$$
\lim _{t \rightarrow+\infty} \mathbb{E}[\psi(u(t))]=\int_{X_{\eta, p}} \psi(J \xi) \mu(\mathrm{d} \xi) .
$$

Proof. Take $u_{0} \in \tilde{X}_{\mu, \delta}$ and define, as usual, $v_{0}=Q u_{0}$. Let $u(t)$ be the solution to problem (1.2) defined by (2.13):

$$
u(t)= \begin{cases}J v(t), & t>0 \\ u_{0}(t), & t \leq 0\end{cases}
$$

Let $\psi \in C_{b}(H)$. Then

$$
\lim _{t \rightarrow+\infty} \mathbb{E}\left[\psi\left(u\left(t ; u_{0}\right)\right)\right]=\lim _{t \rightarrow+\infty} \mathbb{E}\left[\psi\left(J v\left(t ; Q u_{0}\right)\right)\right]=\int_{X_{\eta, p}} \psi(J \xi) \mu(\mathrm{d} \xi)
$$

and the claim follows.

Thus, we claim that system (1.2) shows, under the additional assumptions in Theorem 6.2, an ergodic behaviour, in the sense that for every initial history $u_{0}$, the solution converges in law to a given measure $\mu \# J^{-1}$.

\section{References}

[1] Bonaccorsi, S., Confortola, F., Mastrogiacomo, E.: Optimal control for stochastic volterra equations with completely monotone kernels. SIAM J. Control Optim. (to appear)

[2] Bonaccorsi, S., Desch, W.: Volterra equations perturbed by noise. Technical Report UTM 698, University of Trento (2006)

[3] Bonaccorsi, S., Fantozzi, M.: Large deviation principle for semilinear stochastic Volterra equations. Dyn. Syst. Appl. 13(2), 203-219 (2004)

[4] Bonaccorsi, S., Fantozzi, M.: Infinite dimensional stochastic Volterra equations with dissipative nonlinearity. Dyn. Syst. Appl. 15(3-4), 465-478 (2006)

[5] Bonaccorsi, S., Mastrogiacomo, E.: An analytic approach to stochastic Volterra equations with completely monotone kernels. J. Evol. Equ. 9(2), 315-339 (2009)

[6] Bonaccorsi, S., Tubaro, L.: Mittag-Leffler's function and stochastic linear Volterra equations of convolution type. Stoch. Anal. Appl. 21(1), 61-78 (2003)

[7] Clément, P., Da Prato, G.: Some results on stochastic convolutions arising in Volterra equations perturbed by noise. Atti Accad. Naz. Lincei Cl. Sci. Fis. Mat. Natur. Rend. Lincei (9) Math. Appl. 7(3), 147-153 (1996)

[8] Clément, P., Da Prato, G.: White noise perturbation of the heat equation in materials with memory. Dyn. Syst. Appl. 6(4), 441-460 (1997)

[9] Clément, P., Da Prato, G., Prüss, J.: White noise perturbation of the equations of linear parabolic viscoelasticity. Rend. Istit. Mat. Univ. Trieste 29(1-2):207220 (1998) 1997 
[10] Clément, P., Desch, W., Homan, K.W.: An analytic semigroup setting for a class of Volterra equations. Report No. 202, Special Research Center F 003, Optimization and Control. University of Graz and Technical University of Graz (2002)

[11] Clément, P., Londen, S.-O., Simonett, G.: Quasilinear evolutionary equations and continuous interpolation spaces. J. Differ. Equ. 196, 418-447 (2004)

[12] Da Prato, G., Zabczyk, J.: Stochastic equations in infinite dimensions. Encyclopedia of Mathematics and its Applications, vol. 44. Cambridge University Press, Cambridge (1992)

[13] Desch, W., Londen, S.-O.: On a stochastic parabolic integral equation. In: Functional Analysis and Evolution Equations, pp. 157-169. Birkhäuser, Basel (2008)

[14] Desch, W., Londen, S.-O.: An $L_{p}$-theory for stochastic integral equations. J. Evol. Equ. 11(2), 287-317 (2011)

[15] Desch, W., Miller, R.K.: Exponential stabilization of Volterra integral equations with singular kernels. J. Integral Equ. Appl. 1(3), 397-433 (1988)

[16] Dettweiler, J., Weis, L., van Neerven, J.: Space-time regularity of solutions of the parabolic stochastic Cauchy problem. Stoch. Anal. Appl. 24(4), 843-869 (2006)

[17] Diestel, J., Jarchow, H., Tonge, A.: Absolutely summing operators, Cambridge Studies in Advanced Mathematics, vol. 43. Cambridge University Press, Cambridge (1995)

[18] Gripenberg, G., Londen, S.O., Staffans, O.: Volterra Integral and Functional Equations. Encyclopedia of Mathematics and its Applications. Cambridge University Press, Cambridge (1990)

[19] Haase, M.: The Functional Calculus for Sectorial Operators. Operator Theory: Advances and Applications, vol. 169. Birkhäuser, Basel (2006)

[20] Homan, K.W.: An analytic semigroup approach to convolution Volterra equations. PhD thesis, Technische Universiteit Delft (2003)

[21] Lunardi, A.: Analytic semigroups and optimal regularity in parabolic problems. Progress in Nonlinear Differential Equations and their Applications, vol. 16. Birkhäuser, Basel (1995)

[22] Prüss, J.: Evolutionary integral equations and applications. Monographs in Mathematics, vol. 87. Birkhäuser, Basel (1993)

[23] Rubio de Francia, J.L.: Martingale and integral transforms for Banach space valued functions. In: Probability and Banach Spaces (Zaragoza, 1985). Lecture Notes in Math., vol. 1221, pp. 195-222. Springer, Berlin (1986)

[24] van Neerven, J., Veraar, M.C.: On the stochastic Fubini theorem in infinite dimensions. In: Stochastic Partial Differential Equations and ApplicationsVII. Lecture Notes Pure Appl. Math., vol. 245, pp. 323-336. Chapman and Hall/CRC, Boca Raton (2006) 
[25] van Neerven, J.M.A.M., Veraar, M.C., Weis, L.: Stochastic integration in UMD Banach spaces. Ann. Probab. 35(4), 1438-1478 (2007)

[26] van Neerven, J.M.A.M., Veraar, M.C., Weis, L.: Stochastic evolution equations in UMD Banach spaces. J. Funct. Anal. 255(4), 940-993 (2008)

[27] van Neerven, J.M.A.M., Weis, L.: Invariant measures for the linear stochastic Cauchy problem and $R$-boundedness of the resolvent. J. Evol. Equ. 6(2), 205-228 (2006)

[28] van Neerven, J.M.A.M., Weis, L.: Stochastic integration of operator-valued functions with respect to Banach space-valued Brownian motion. Potential Anal. 29(1), 65-88 (2008)

[29] Zacher, R.: Quasilinear parabolic problems with nonlinear boundary conditions. Dissertation, Martin-Luther-Universität Halle-Wittenberg (2003)

Stefano Bonaccorsi

Dipartimento di Matematica

Università di Trento

Via Sommarive 14

38123 Povo

Trento

Italy

e-mail: stefano.bonaccorsi@unitn.it

Gertrud Desch

Institut für Mathematik und Wissenschaftliches Rechnen

Karl-Franzens-Universität Graz

Heinrichstrasse 36

8010 Graz

Austria

e-mail: gertrud.desch@uni-graz.at

Received: 20 July 2010.

Accepted: 23 March 2012. 